\title{
ARTICLE OPEN \\ Influenza- and MCMV-induced memory CD8 T cells control respiratory vaccinia virus infection despite residence in distinct anatomical niches
}

\author{
Suzanne P. M. Welten ${ }^{1}$, Josua Oderbolz ${ }^{1}$, Vural Yilmaz ${ }^{1}$, Susanna R. Bidgood ${ }^{2}$, Victoria Gould ${ }^{2}$, Jason Mercer ${ }^{2,3}$, Roman Spörri ${ }^{1}$ and \\ Annette Oxenius (iD)
}

\begin{abstract}
Induction of memory CD8 T cells residing in peripheral tissues is of interest for T cell-based vaccines as these cells are located at mucosal and barrier sites and can immediately exert effector functions, thus providing protection in case of local pathogen encounter. Different memory CD8 T cell subsets patrol peripheral tissues, but it is unclear which subset is superior in providing protection upon secondary infections. We used influenza virus to induce predominantly tissue resident memory $\mathrm{T}$ cells or cytomegalovirus to elicit a large pool of effector-like memory cells in the lungs and determined their early protective capacity and mechanism of reactivation. Both memory CD8 T cell pools have unique characteristics with respect to their phenotype, localization, and maintenance. However, these distinct features do not translate into different capacities to control a respiratory vaccinia virus challenge in an antigen-specific manner, although differential activation mechanisms are utilized. While influenza-induced memory CD8 T cells respond to antigen by local proliferation, MCMV-induced memory CD8 T cells relocate from the vasculature into the tissue in an antigen-independent and partially chemokine-driven manner. Together these results bear relevance for the development of vaccines aimed at eliciting a protective memory CD8 T cell pool at mucosal sites.
\end{abstract}

Mucosal Immunology (2021) 14:728-742; https://doi.org/10.1038/s41385-020-00373-4

\section{INTRODUCTION}

CD8 T cells are activated in an antigen-specific manner and have the ability to eliminate pathogens by producing effector cytokines and exerting cytotoxic functions. Upon viral infection, naive virus-specific CD8 T cells are activated, clonally expand, and give rise to a pool of effector cells capable of killing infected target cells. A small population of T cells persists as memory cells that have the capacity to respond and rapidly expand upon secondary antigen encounter. These long-lasting memory CD8 $\mathrm{T}$ cells are the basis for $\mathrm{T}$ cell-based vaccination approaches. Memory $T$ cells form a heterogeneous population, where distinct subsets are defined based on differences in cell surface molecules, anatomical localization, proliferation capacity, effector functions and metabolism. ${ }^{1}$

Central memory $T$ cells $\left(T_{C M}\right)$ express markers that permit lymph node homing and are therefore predominantly found in secondary lymphoid tissues, but these cells also recirculate. In addition, $\mathrm{T}_{\mathrm{CM}}$ cells exhibit profound proliferative potential. Effector memory $T$ cells $\left(T_{E M}\right)$ mainly recirculate and do not express lymphoid tissue homing molecules. One hallmark of these cells is their robust effector functions. Although both $T_{E M}$ and $T_{C M}$ recirculate in the vasculature, it is thought that the reactivation of $\mathrm{T}_{\mathrm{CM}}$ cells is not immediate. Antigen first has to be transported to the lymphoid tissues where it is presented by professional antigen presenting cells to $T$ cell zone-homing and resident $T_{C M}$ cells. It is not entirely clear how $\mathrm{T}_{E M}$ cells are reactivated, but there is evidence that the size of the $T_{E M}$ pool in peripheral tissues and blood is directly linked to its early protective capacity, ${ }^{2-4}$ indicating that these cells respond directly in the infected tissue. Circulatory memory $\mathrm{T}$ cells can also be divided into distinct subsets using the expression of the fractalkine receptor CX3CR1., Tissue resident memory $\left(\mathrm{T}_{\mathrm{RM}}\right) \mathrm{T}$ cells are another subset of memory $\mathrm{T}$ cells, lodged in peripheral tissues, such as the lungs, salivary gland, gut, female reproductive tract, and the skin. ${ }^{7-12}$ In contrast to $T_{E M}$ cells, $T_{R M}$ cells are restricted from the circulation and are transcriptionally distinct from circulatory memory T cells. ${ }^{13}$ The initial signals that induce this phenotype depend on tissuespecific cues and include TGF- $\beta$, IL-15, and local antigen. ${ }^{13} \mathrm{~T}_{\mathrm{RM}}$ cells rapidly exert their effector functions upon antigen encounter, leading to an anti-viral state in the tissue and to the attraction of other immune cells to the site of inflammation. ${ }^{14,15}$ Thus, memory $T$ cells residing in peripheral tissues are poised for instant action and are located at barrier sites, which is where pathogens enter the body. Due to these characteristics, both $T_{R M}$ and $T_{E M}$ cells have gained interest in being exploited for vaccination purposes. However, it is not clear which T cell subset is superior in providing early protection upon secondary challenge in peripheral tissues.

In order to induce large numbers of effector-like $T$ cells in peripheral tissues, cytomegalovirus (CMV)-based vectors are an interesting option. CMV infection induces an atypical CD8 T cell response, characterized by the accumulation of large numbers of effector-like $T$ cells in the circulation, a process termed memory

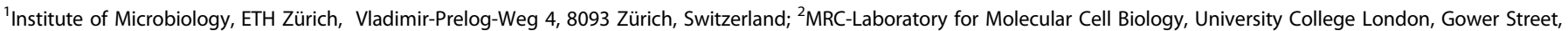
London WC1E 6BT, UK and ${ }^{3}$ Institute of Microbiology and Infection, University of Birmingham, Edgbaston, Birmingham B15 2TT, UK

Correspondence: Annette Oxenius (aoxenius@micro.biol.ethz.ch)

Received: 24 July 2020 Revised: 25 November 2020 Accepted: 14 December 2020

Published online: 21 January 2021 
inflation., 46,17 Maintenance of the inflationary $\mathrm{T}$ cell pool is dependent on antigen presentation by latently infected nonhematopoietic cells. ${ }^{18}$ Epitopes that induce inflationary $\mathrm{T}$ cell responses are processed by the constitutive proteasome, and this pathway can be utilized by inserting the epitope on the Cterminus of a gene of interest in the viral genome. ${ }^{19,20}$ In preclinical animal models, CMV-based vectors encoding foreign antigens derived from tumors or pathogens induced effectorlike $T$ cell responses specific for these inserted epitopes. These T cells provided protection from the relevant tumors or pathogens expressing these epitopes. ${ }^{21-29}$ The protective capacity correlated with the size of the inflationary $\mathrm{T}$ cell pool in the peripheral organ and blood. , $3,29^{2}$

Here, we compared the local protective capacity of antigenicspecific CD8 $T_{R M}$ cells (induced by influenza virus infection) and inflationary $T$ cells (induced by MCMV infection) in lung tissue towards a secondary respiratory infection with vaccinia virus (VV) encoding the same antigen. We found that both influenza- and MCMV-induced memory $T$ cells provided protection from a local VV challenge in an antigen-dependent fashion. However, the manner of the response was distinct. Whereas influenza-induced memory $\mathrm{T}$ cells increased the local pool of $\mathrm{T}$ cells by local proliferation and T cell recruitment, the MCMV-induced T cell pool migrated out of the vasculature into the lung parenchyma, which was partially driven by chemokines, enabling these cells to exert their effector functions at the site of infection.

\section{RESULTS}

The phenotypic composition of the memory $T$ cell pool in the lungs is virus dependent

We established a model to compare the immediate protective capacity of distinct memory T cell pools in a peripheral tissue. We focused on the lungs as both $\mathrm{T}_{\mathrm{RM}}$ cells and MCMV-induced inflationary $T$ cells are established in this tissue. ${ }^{30-32}$ To compare memory T cells with the same specificity, naive CD45.1 $1^{+} \mathrm{TCR}$ transgenic OVA $257-264 / \mathrm{K}^{\mathrm{b}}$-specific (OT-I) $\mathrm{T}$ cells were adoptively transferred into CD45.2 ${ }^{+}$WT recipients before infection. Mice were then intra-tracheally (i.t.) infected with an influenza strain expressing the $\mathrm{OVA}_{257-264}$ epitope, PR8-OVA (Fig. 1a). A large pool of $T_{R M}$ cells was induced, as the majority of the OT-I T cells in the lungs expressed the typical $T_{R M}$ markers CD69 and CD103 four weeks post infection (Fig. $1 \mathrm{~b}, \mathrm{c}$ ), but no sizeable $\mathrm{KLRG}^{+}$ population was detected. To induce a large pool of effector-like cells in the lungs, mice were systemically infected with an MCMVvector expressing the $\mathrm{OVA}_{257-264} / \mathrm{K}^{\mathrm{b}}$ epitope under the control of the ie2 promoter (MCMV-ie2-SIINFEKL), inducing an OVA $257-264 / \mathrm{K}^{\mathrm{b}}$ specific $T$ cell response with inflationary $T$ cell characteristics. ${ }^{2,19}$ The majority of the lung OT-I T cells expressed KLRG1, whereas hardly any expressed CD69 and CD103 (Fig. 1b, c), consistent with the effector-like phenotype of the MCMV-induced inflationary memory population. Similar results were found when endogenous $\mathrm{OVA}_{257-264} / \mathrm{K}^{\mathrm{b}}$-specific CD8 T cells were analyzed instead of adoptively transferred TCR-transgenic OT-I cells (Supplementary Fig. 1A, B). In both influenza-induced and MCMV-induced memory CD8 T cells, a proportion expressed interleukin 7 receptor subunit alpha (IL7Ra, CD127). For influenza-induced OT-I T cells, this marker was mostly expressed by the $\mathrm{T}_{\mathrm{RM}} \mathrm{CD} 69^{+} \mathrm{CD} 103^{+}$cells (Fig. 1d). In both settings, few OT-I T cells co-expressed CD127 and CD62L, representing $T_{C M}$ cells (Fig. 1e). Although administration of MCMV via the respiratory route was reported to induce MCMVspecific $T_{R M}$ cells in the lungs, ${ }^{33-35}$ in our experimental setup both i.t. and systemic administration of MCMV predominantly induced an effector-like population of MCMV-specific CD8 T cells, and i.t. administration of the virus only slightly increased the proportion of $T_{R M}$ cells (Supplementary Fig. 2A, B). This discrepancy might be explained by differences in genetics of the used mouse strains. We therefore henceforth used i.t. influenza virus infection to induce a
CD8 $T_{R M}$ population and systemic MCMV infection to raise an effector-like CD8 memory population.

$\mathrm{T}_{\mathrm{RM}}$ cells are restricted from the circulation and located deep within tissues. To discriminate between cells within the tissue and those within/close to the vasculature, a fluorescently conjugated aCD8 antibody was i.v. injected into mice shortly before euthanasia. As expected, the majority of influenza-induced memory OT-I T cells was not labeled by this procedure, whereas MCMV-induced OT-I T cells stained mostly positive for the i.v. injected antibody, indicating that these cells are within or close to the vasculature (Fig. 1f). Similar results were observed for endogenous $\mathrm{OVA}_{257-264} / \mathrm{K}^{\mathrm{b}}$-specific CD8 T cells (Supplementary Fig. 1C). The distinct localizations of the memory OT-I T cells were confirmed by microscopy. Influenza-induced memory OT-I T cells were located in close proximity to Epcam ${ }^{+}$epithelial cells lining the lung airways and were not labeled by the i.v. injected aCD8 antibody. In contrast, MCMV-induced OT-I T cells were found in close proximity to $\mathrm{CD} 31^{+}$vascular endothelial cells and labeled by the short intravascular exposure to the aCD8 antibody (Fig. 1g).

In addition, we evaluated the phenotype of MCMV- and influenza-specific OT-I memory T cells using T-distributed stochastic neighbor embedding (t-SNE) analysis and observed heterogeneity between the different memory $\mathrm{T}$ cell pools (Fig. 1h). Besides phenotypical differences, the magnitude of the lung memory OT-I T cell pool was also different, as four times more OT-I T cells were detected in the MCMV setting (Fig. 1i). The number of MCMV-specific OT-I T cells in the lungs did not change drastically over a time period of 60 days. In contrast, 60 days post influenza virus infection, a $\sim 6$-fold reduction in the number of OT-I T cells in the lungs was observed (Fig. 1i). The $T_{R M}$ cells in particular were declining in the lung tissue, as the percentage of $\mathrm{CD} 69^{+} \mathrm{CD} 103^{+}$ OT-I T cells had dropped considerably at 60 days post influenza virus infection (Fig. 1j). This is in agreement with previous studies showing that $T_{\mathrm{RM}}$ cells in the lung are not steadily maintained. ${ }^{36,37}$ These findings were confirmed for endogenous $\mathrm{OVA}_{257-264^{-}}$ specific CD8 T cells (Supplementary Fig. 1D). The decrease in OT-I T cells in influenza infection was not observed in the spleen (Supplementary Fig. 1E). Combined, these results show that influenza virus and MCMV infection induce memory CD8 T cell populations in the lungs with distinct features in terms of their phenotype, localization, and maintenance.

T cells induced by MCMV or influenza virus exhibit comparable protective capacity

To address the question whether the protective capacity of the distinct memory $\mathrm{T}$ cell compositions in the lungs differ, we performed a challenge infection with a recombinant $\mathrm{V}$ expressing the shared $\mathrm{OVA}_{257-264}$ antigen (VV-OVA). Specifically, influenza- or MCMV-experienced mice were exposed to a local (i.t.) VV-OVA infection and the viral load in the lungs was determined 2 days later (Fig. 2a). A group of naive mice was included that harbored no pulmonary memory $\mathrm{OVA}_{257-264} / \mathrm{K}^{\mathrm{b}}$ specific $T$ cells before the VV-OVA challenge. Both groups of memory mice showed enhanced control of a local respiratory $\mathrm{VV}$ challenge as compared to naive mice (Fig. 2b). However, no differences in the viral load were observed between influenza- or MCMV-experienced mice. This result indicates that the distinct memory CD8 T cell compositions protect equally well from VOVA infection, irrespective if the majority of the memory $T$ cell pool harbored $T_{R M}$ or effector-like cells. Examining the protective capacity of endogenous $\mathrm{OVA}_{257-264} / \mathrm{K}^{\mathrm{b}}$-specific memory CD8 $T$ cells revealed comparable results (Supplementary Fig. 3 ). The protective effect of the memory OT-I T cells was antigen dependent, as challenge with a V that did not express the cognate shared antigen was not controlled above naive mice (Fig. $2 \mathrm{c}$ ). Since the number of $\mathrm{T}_{\mathrm{RM}}$ cells in the lungs of influenza virus infected mice declined in time, mice were challenged with VV-OVA at a later time point to determine if the protective 

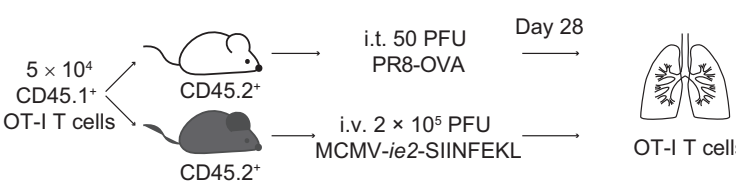

C $\square$ Influenza (PR8-OVA) $=$ MCMV-ie2-SIINFEKL
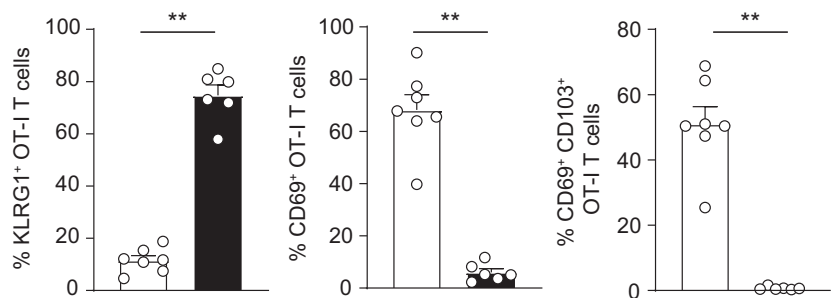

d
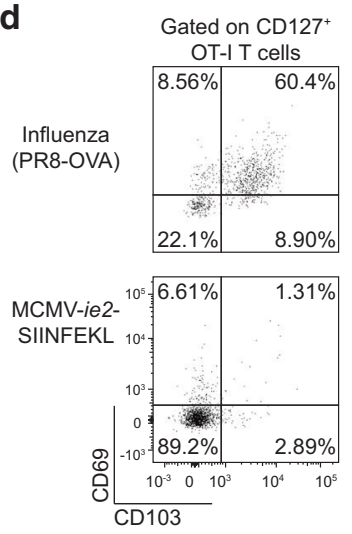

e
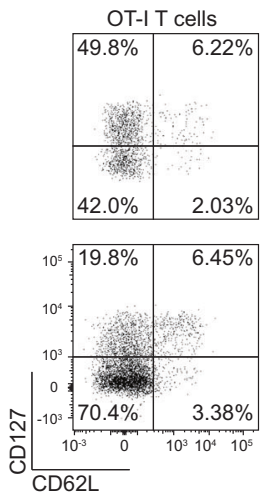

g
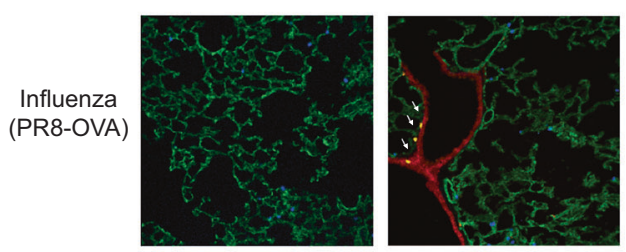

MCMV-ie2-
SIINFEKL
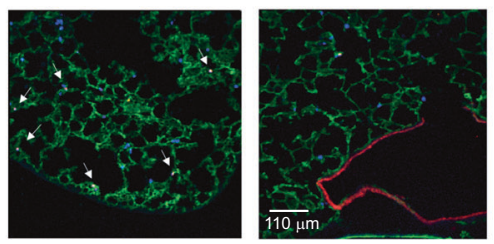

CD31

CD8 (i.v.) Epcam

CD45.1

i
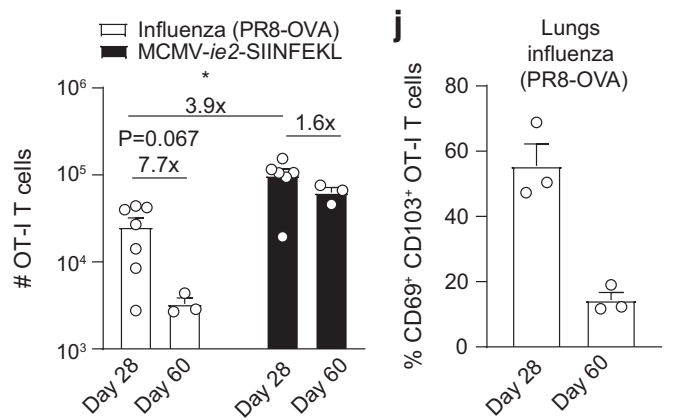

Day 28 lungs
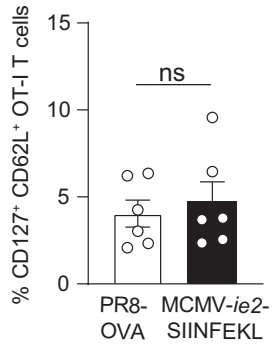

h

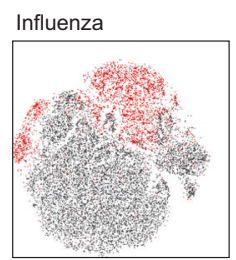

CD127- KLRG1+

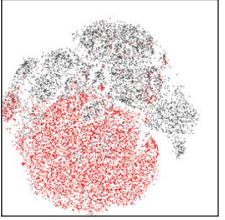

$\mathrm{CD} 69+\mathrm{CD} 103$
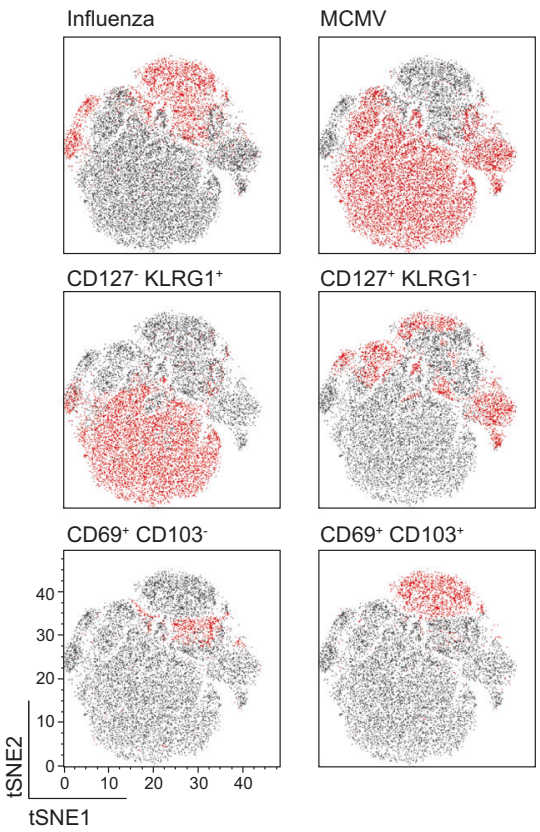

$\mathrm{CD}_{127^{+} \mathrm{KLRG} 1}$

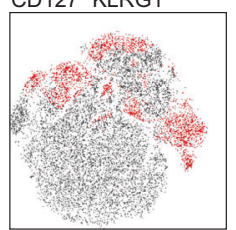

$\mathrm{CD}^{2} 9^{+} \mathrm{CD} 103^{+}$
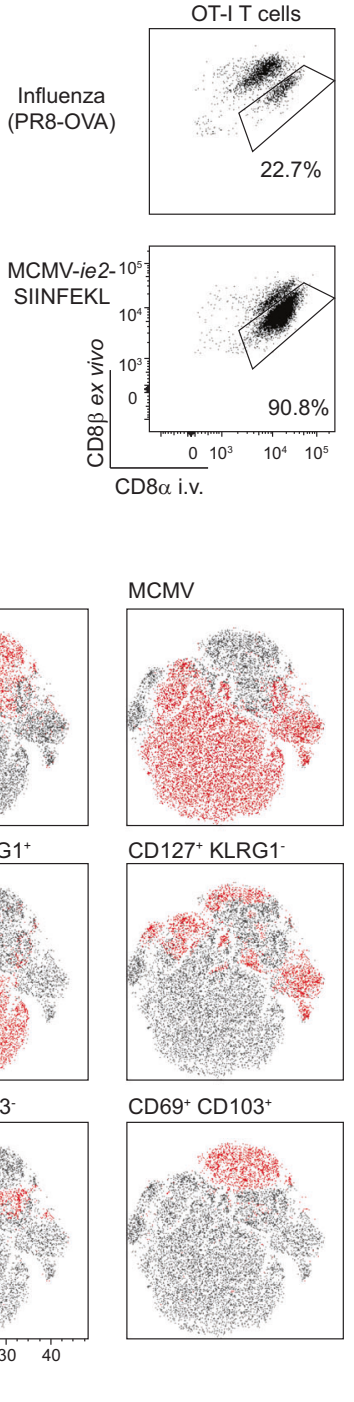
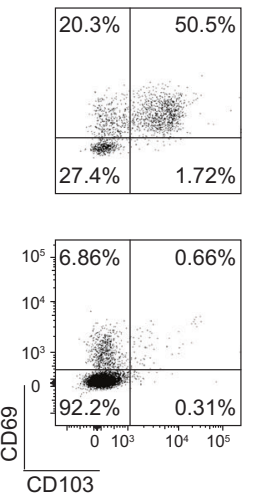

$\overline{\mathrm{CD} 103}$

f

Day 28 lungs

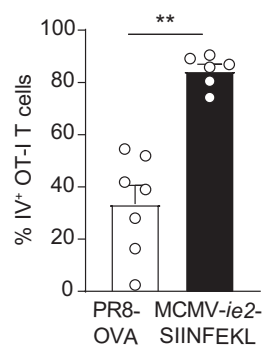

CD8 $\alpha$ i.v. ${ }^{+}$

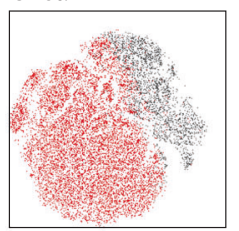

CX3CR1 $1^{+}$

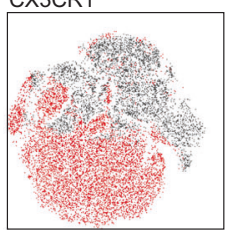

$\mathrm{CD}^{2} \mathrm{~L}^{+}$

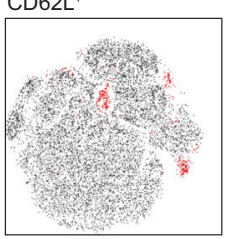

capacity would also diminish compared to MCMV-primed mice. However, despite the reduction in $T_{R M}$ cells, mice that had memory $T$ cells in the lungs, irrespective of whether they were induced by influenza virus or MCMV, showed enhanced control of
VV-OVA challenge (Fig. 2d). These results show that mice with memory CD8 T cells in the lung tissue exhibit enhanced control from a local VV rechallenge, irrespective of the phenotypic memory $\mathrm{T}$ cell composition. 
Fig. 1 MCMV and influenza infection induce distinct memory CD8 $\mathbf{T}$ cell subsets in the lungs. a Experimental setup: $5 \times 10^{4} \mathrm{CD} 45.1^{+} \mathrm{OT}-\mathrm{I}$

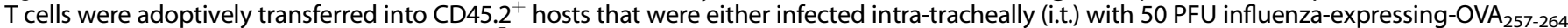
(PR8-OVA) or intravenously (i.v.) with $2 \times 10^{5}$ PFU MCMV-expressing-OVA $257-264$ (MCMV-ie2-SIINFEKL). Twenty-eight days post infection, the phenotype of the OT-I T cells in the lungs was determined. b Representative flow cytometry plots show the cell surface expression of CD127 KLRG1 and of CD69 CD103 on OT-I T cells in the lungs. c Bar graphs show the percentage of OT-I T cells that expresses KLRG1, CD69 or CD69 CD103, indicated as mean + SEM. Data is pooled from two independent experiments $(n=6-7$, each dot represents an individual mouse). d Flow cytometry plots show expression of CD69 CD103 on CD127 $7^{+}$OT-I T cells in the lungs 28 days post infection. e Flow cytometry plots show expression of CD127 CD62L on OT-I T cells in the lungs 28 days post infection. Bar graph shows the percentage of CD127 ${ }^{+}$CD62L ${ }^{+}$OT-I T cells as mean + SEM. Data is pooled from two independent experiments ( $n=6$, each dot represents an individual mouse). $f$ Mice received $5 \mu \mathrm{g}$ fluorescently conjugated $\alpha \mathrm{CD} 8$ antibody 3 min prior to euthanasia. Flow cytometry plots show in vivo CD8 $\alpha$ labeling and ex vivo CD8 $\beta$ staining in the lungs, 28 days post-infection on OT-I T cells. Bar graphs indicate the percentage of OT-I T cells stained by the i.v. injected CD8 antibody as mean + SEM. Data is pooled from two independent experiments ( $n=6-7$, each dot represents an individual mouse). $g$ Microscopy image shows the lung tissue 28 days after infection. Arrows indicate OT-I T cells. $\mathbf{h}$ tSNE clusters of CD45.1 $1^{+}$OT-I T cells isolated from the lungs of influenza- or MCMV-infected mice, based on expression of CD44, CD8 $\alpha$ i.v., CD127, KLRG1, CX3CR1, CD69, CD103, and CD62L. Expression of each marker is color-coded with red = expressed and black $=$ not expressed $(n=4$ mice per group). $\mathbf{i}$ The total number of OT-I T cells recovered from the lungs is shown at day 28 and day 60 post-infection $(n=3-7)$. Fold difference is indicated. $\mathbf{j}$ The percentage of OT-I T cells in influenza virus infection that expresses CD69 CD103 is shown on day 28 and 60 post-infection, one experiment out of two is shown ( $n=3$ ), as mean + SEM, each dot represents an individual mouse. Two-sided Mann-Whitney test was used to determine statistical significance: ${ }^{*} P<0.05$, ${ }^{* *} P<0.01$ and not significant (ns) $P \geq 0.05$.

a
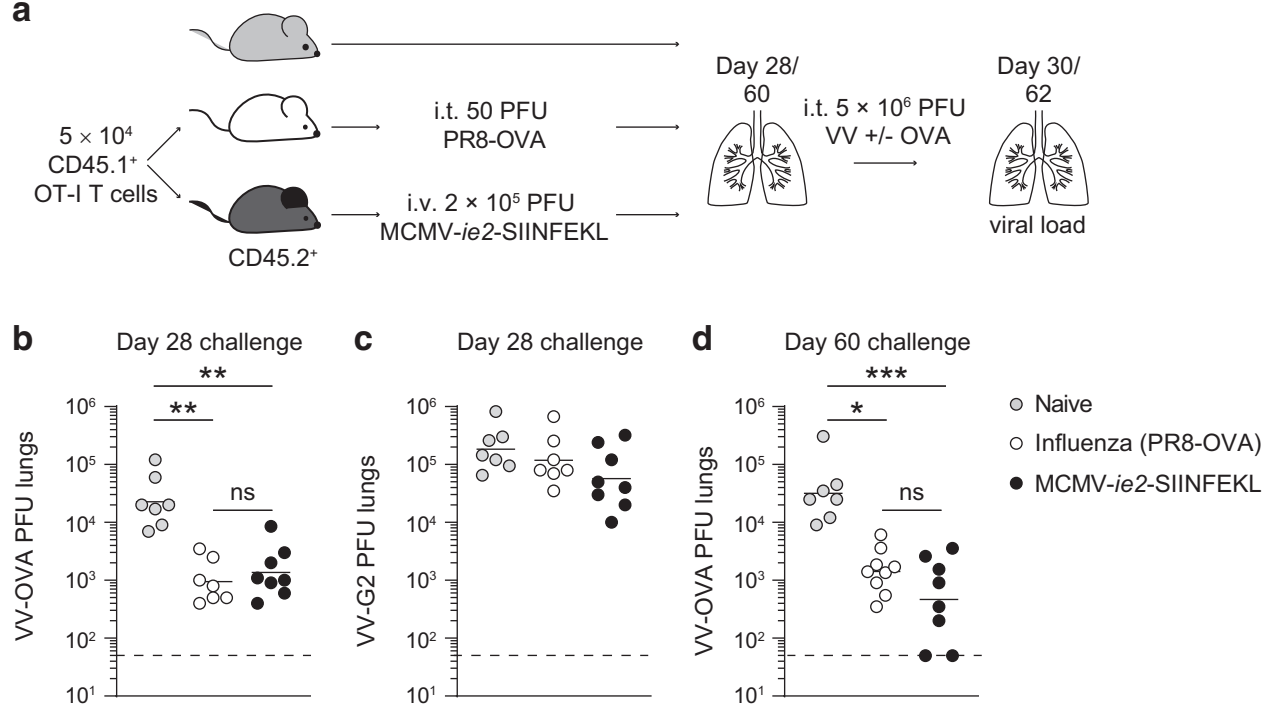

Fig. 2 MCMV- and influenza-induced memory $T$ cells protect from a local vaccinia virus challenge in the lungs in an antigen-specific manner. a Experimental setup: mice with memory T cells in the lungs induced by influenza virus or MCMV (as described in Fig. $1 \mathrm{a}$ ) were challenged i.t. on day $28(\mathbf{b}, \mathbf{c})$, or day 60 (d) with $5 \times 10^{6}$ PFU vaccinia virus expressing OVA (b, d) or with $3 \times 10^{5}$ PFU VV-G2 (vaccinia virus that does not express OVA) (c). Two days post-challenge the viral load was determined in the lungs. All data is pooled from two independent experiments $(n=3-5)$. Each dot represents an individual mouse, geometric mean and limit of detection are indicated. Statistical significance was determined using one-way ANOVA with Kruskal-Wallis post hoc test to correct for multiple comparisons: ${ }^{*} P<0.05,{ }^{* *} P<0.01,{ }^{* * *} P<0.001$ and not significant (ns) $P \geq 0.05$.

T cells induced by influenza virus or MCMV infection produce effector cytokines

Memory CD8 T cells in the lungs provided protection from local challenge in an antigen-dependent manner. As the memory OT-I cells induced by influenza virus or MCMV infection had different characteristics, we next addressed whether the mechanism of responding to antigenic challenge would differ. IFN- $\gamma$ and TNF produced by CD8 $T$ cells are critical for the clearance of a respiratory $\mathrm{V}$ infection. ${ }^{38,39}$ We examined the quality of the responding memory $T$ cells by examining cytokine production before ( 28 days post primary infection) and 2 days post-secondary challenge with VV-OVA. OT-I T cells produced IFN- $\gamma$, TNF, and IL-2 (Fig. 3a), independent of which virus was initially used for the establishment of the memory pool. Also, after WV-OVA challenge, the majority of cells in both settings was able to produce these effector cytokines (Fig. 3b-d). To conclude, memory CD8 T cell populations induced in the lungs by either MCMV or influenza virus are both capable of producing effector cytokines upon secondary exposure to cognate antigen.
The lung OT-I T cell pool increases in size upon antigenic rechallenge

Infection with MCMV-ie2-SIINFEKL induced a memory OT-I T cell pool in the lungs that was higher in magnitude as compared to the $T_{R M}$ pool induced upon influenza (PR8-OVA) virus infection (Fig. 1i). Next, we addressed the size of the OT-I T cell pool 2 days post challenge with VV-OVA. In both the MCMV and influenza virus setting, the OT-I memory $T$ cell population increased in magnitude after local antigen re-exposure upon VV-OVA infection (Fig. 4a). Although the overall expansion of the OT-I T cell pool in the lung was more pronounced in the influenza virus setting (3.6fold versus 2 -fold), the population was still 2-fold larger in the MCMV setting. Also, the number of cytokine-producing cells was larger in the MCMV setting (Supplementary Fig. 4A-C). In contrast to the lung, no changes in the magnitude of the OT-I T cell pool were detected in the influenza virus setting in the spleen (Fig. 4b). Strikingly, in the MCMV setting, splenic OT-I T cells were reduced upon i.t. VV-OVA infection (Fig. 4c), likely indicating that splenic OT-I cells migrated and accumulated in the lung tissue. To 
a

Lungs, 2 days post VV-OVA
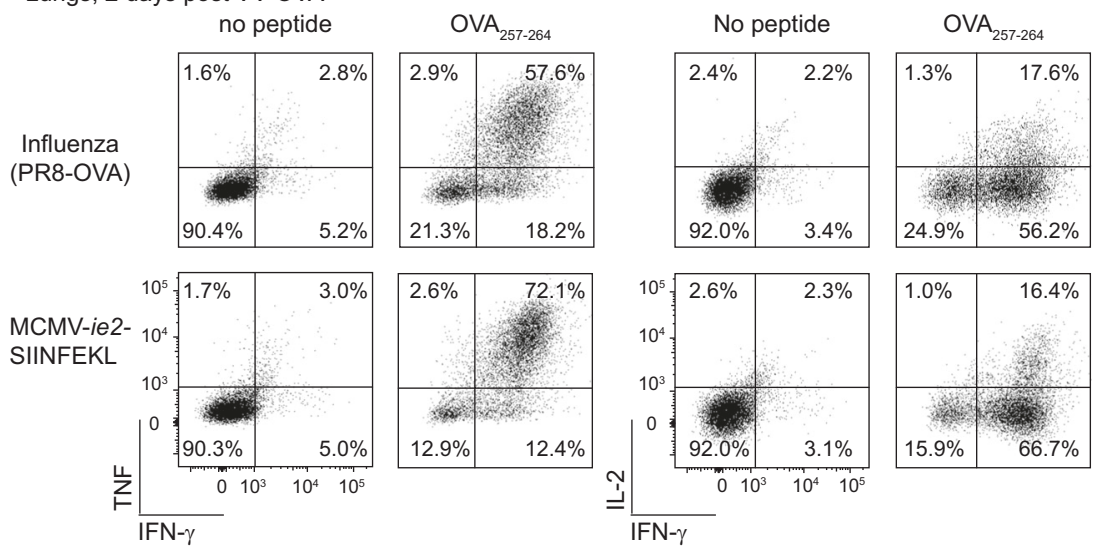

b
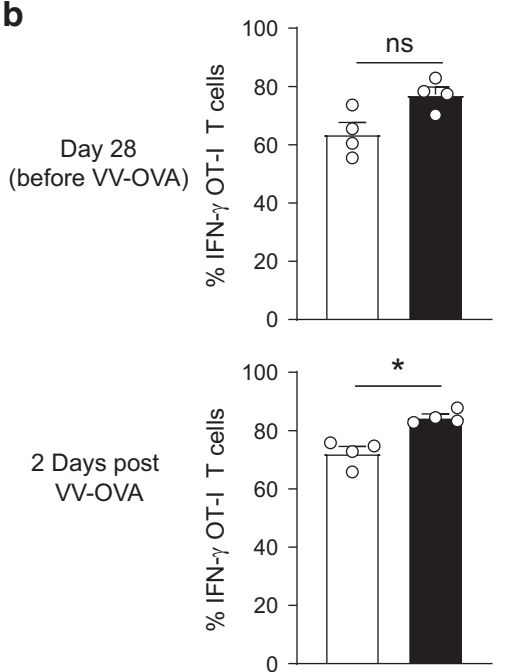

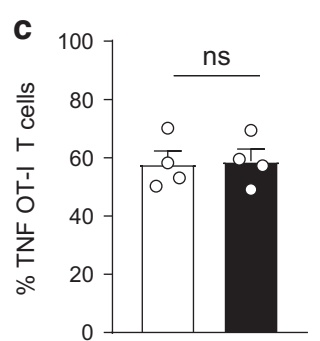

d

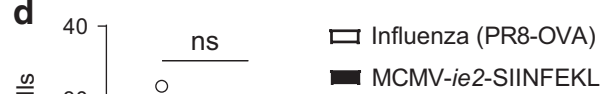

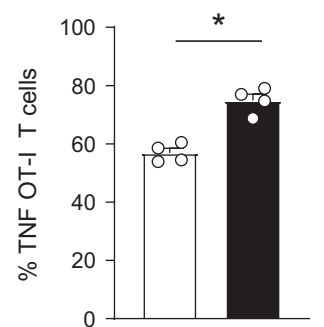

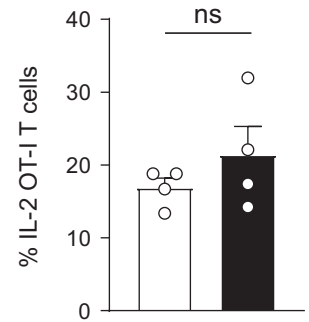

Fig. 3 Memory T cells induced by MCMV or influenza virus produce effector cytokines. Cytokine production was determined in OT-I T cells from the lungs of MCMV-infected or influenza virus-infected mice, before (28 days post primary infection, top row) and 2 days post (bottom row) challenge with $5 \times 10^{6}$ PFU VV-OVA. a Representative plots show intracellular production of IFN- $\gamma$ versus TNF (left) and IL-2 (right). b-d Bar graphs show the percentage of OT-I T cells that produces IFN- $\gamma(\mathbf{b})$, TNF (c), or IL-2 (d). All bar graphs represent mean + SEM and each dot represents an individual mouse. One experiment out of two independent experiments is shown. Mann-Whitney test was used to determine significance: ${ }^{*} P<0.05(n=4)$ and not significant (ns) $P \geq 0.05$.

corroborate the importance of $\mathrm{T}$ cell recruitment for the expansion of the lung localized OT-I T cell pool during VV-OVA challenge, mice were treated with FTY720 which inhibits S1P-S1PR-axismediated lymphocyte egress from lymphoid organs. In both the influenza- and MCMV-setting, the lung localized OT-I T cell pool was reduced compared to the untreated group, indicating that $\mathrm{T}$ cell recruitment was contributing to the expansion of the lunglocalized OT-I T cell pool (Supplementary Fig. 4D).

Another explanation for the increase in magnitude of the OT-I $\mathrm{T}$ cells localized in the lung could be local proliferation. We therefore determined Ki67 expression in OT-I T cells to identify cycling cells. A higher percentage of OT-I T cells in the lungs expressed Ki67 in the influenza virus setting after local VV-OVA challenge (Fig. 4d, e), although Ki67 was also expressed in a small population of MCMV-induced OT-I T cells. To corroborate these findings, mice were administered with BrdU in the drinking water during the recall response to determine active DNA synthesis and proliferation. Relatively more OT-I T cells induced by influenza virus infection incorporated BrdU compared to the MCMV setting upon VV-OVA challenge (Fig. 4f, g). In summary, these data show that lung-localized memory $T$ cells induced by influenza virus or MCMV infection expand upon local viral rechallenge. However, a higher proliferative capacity was found in memory T cells induced by influenza virus infection. Together, these results suggest that the increased number of OVA-specific CD8 T cells in the MCMVsetting after local VV-OVA challenge might be primarily due to relocation of circulating cells to the lung tissue.

Memory CD8 T cells induced by MCMV virus infection relocate from the vasculature upon local inflammation

Next, we addressed if the localization of the memory OT-I T cells in the lungs would change upon challenge with a viral infection sharing the cognate antigen. As shown before, the majority of the MCMV-induced OT-I cells in the lungs was close to or within the vasculature (Figs. 1d, 5a). However, after local VV-OVA administration, a large fraction of the OT-I T cells migrated out of the vasculature deeper into the lung parenchyma, as determined by the absence of binding by an i.v. injected, fluorescently conjugated aCD8 antibody (Fig. 5a). Extravasation of OT-I T cells was also observed upon local challenge with a $\mathrm{V}$ not expressing the OVA antigen (VV-G2), indicating that antigen was not a prerequisite for the relocation to occur (Fig. 5b). Moreover, when VV-OVA was administrated i.p. instead of i.t., $\mathrm{OVA}_{257-264} / \mathrm{K}^{\mathrm{b}}$ specific CD8 $T$ cells in the lung did not relocate deeper into the tissue. However, in the ovaries, which is the organ where VV replicates upon an i.p. infection, $\mathrm{OVA}_{257-264} / \mathrm{K}^{\mathrm{b}}$-specific CD8 T cells 

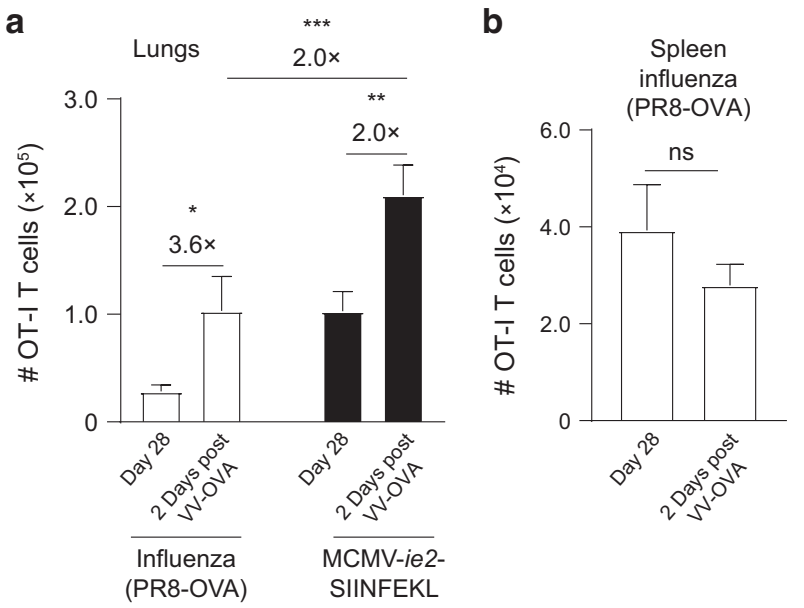

C

$$
\begin{aligned}
& \text { Spleen } \\
& \text { MCMV-ie2-SIINFEKL }
\end{aligned}
$$

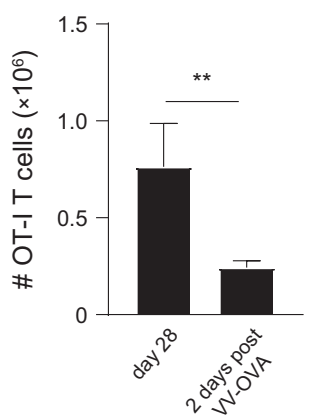

d
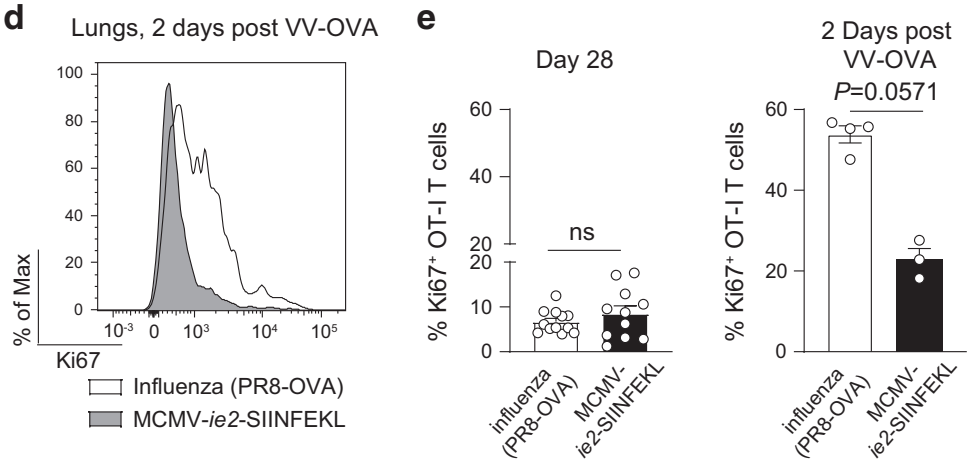

f
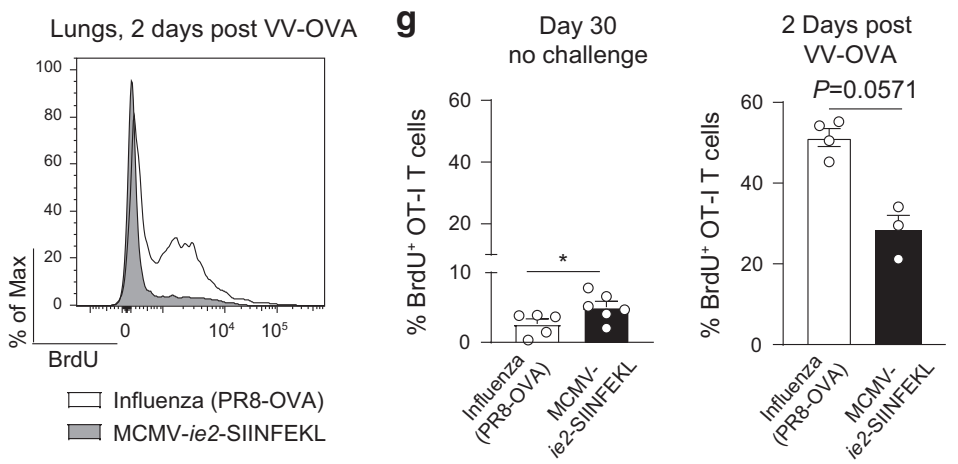

Fig. 4 Memory $\mathrm{T}$ cells in the lung expand upon local rechallenge. Mice with memory OT-I T cells induced by influenza virus (PR8-OVA) or MCMV-ie2-SIINFEKL (as described in Fig. 1A) were i.t. challenged on day 28 with $5 \times 10^{6}$ PFU VV-OVA. a The number of OT-I T cells in the lungs was determined 28 days post-priming and 2 days post-secondary challenge with VV-OVA $(n=18-31$, pooled from eight independent experiments). b Number of OT-I T cells in the spleen 28 days post influenza virus (PR8-OVA) infection and 2 days post-secondary challenge with VV-OVA ( $n=20-26$, pooled from eight independent experiments). c Number of splenic OT-I T cells 28 days post MCMV-ie2-SIINFEKL infection and 2 days post-secondary challenge with VV-OVA ( $n=16-31$, pooled from eight independent experiments). d Histogram shows the expression of Ki67 in lung OT-I T cells 2 days post VV-OVA infection. e Bar graphs show the percentage of Ki67 ${ }^{+}$within OT-I T cells on day 28 post-primary infection (left) ( $n=11-12$, pooled from five independent experiments) or 2 days post-secondary infection with VV-OVA (right) ( $n=3-4$, one experiment out of eight is shown). f Mice received BrdU in the drinking water during the VV-OVA rechallenge period. Histogram shows BrdU incorporation in OT-I T cells in the lungs. g Bar graphs show the percentage of OT-I T cells that incorporated BrdU in mice from day 28 to day 30 (left) ( $n=5-6$, pooled from two independent experiments) and in mice that received a VV-OVA challenge on day 28 (right) ( $n=3-4$, one experiment out of 4 is shown). All bar graphs represent mean + SEM, each dot represents an individual mouse. Two-sided Mann-Whitney test was used to determine statistical significance: ${ }^{*} P<0.05,{ }^{* *} P<0.01,{ }^{* *} P<0.001$ and not significant (ns) $P \geq 0.05$.

extravasated (Supplementary Fig. 5A). Combined these results indicate that local inflammation is required for the extravasation of MCMV-induced memory T cells into the site of the infected tissue.

For memory $T$ cells induced by influenza virus infection and responding to a VV-OVA challenge, we also observed a decrease in the percentage of cells that was stained by the i.v.-injected antibody (i.e. identifying cells within or close to the vasculature). This effect was antigen dependent, as it was not observed upon challenge with VV-G2 (Fig. 5b), likely reflecting an increase of cells in the lung tissue due to local proliferation of the lung localized cells. ${ }^{40,41}$ Microscopy analysis of lung sections showed relocalization of the MCMV-induced OT-I $\mathrm{T}$ cells from $\mathrm{CD}_{3} 1^{+}$endothelial cells closer to the Epcam ${ }^{+}$ epithelial cells lining the airways. These cells were not labeled by the i.v. injected fluorescently conjugated aCD8 antibody, 


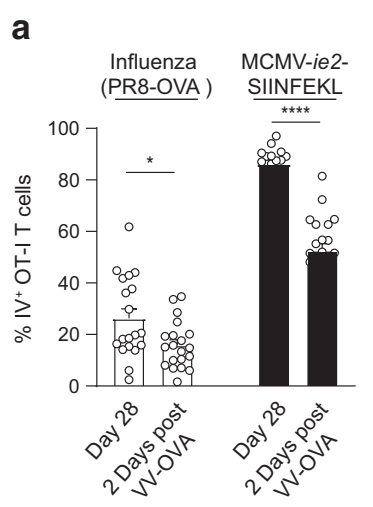

d

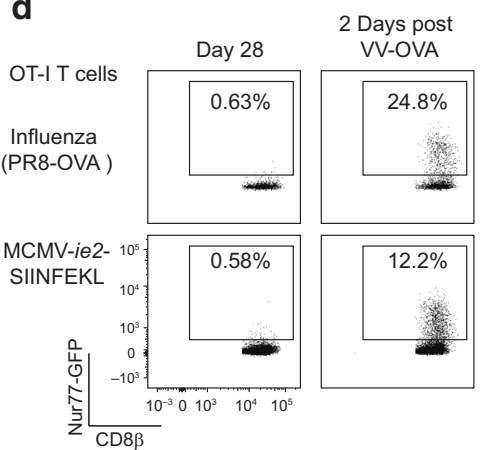

f

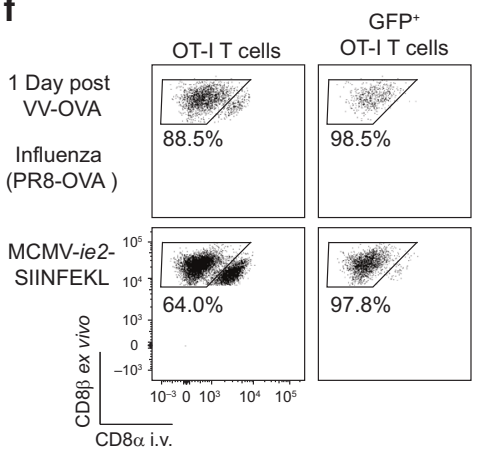

b

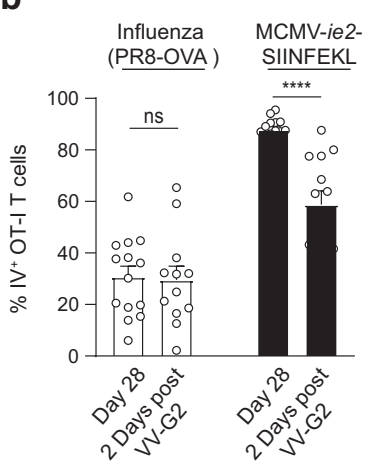

C 2 Days post VV-OVA infection influenza (PR8-OVA)

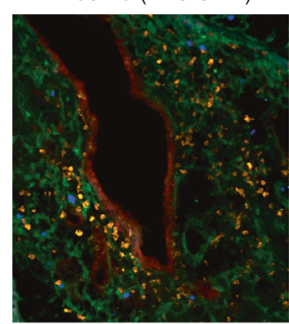

CD31

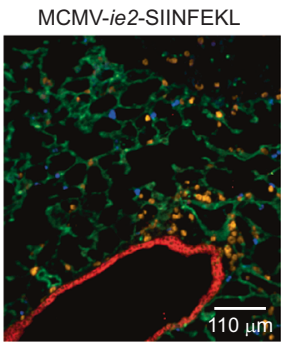

CD8 (i.v.) Epcam e

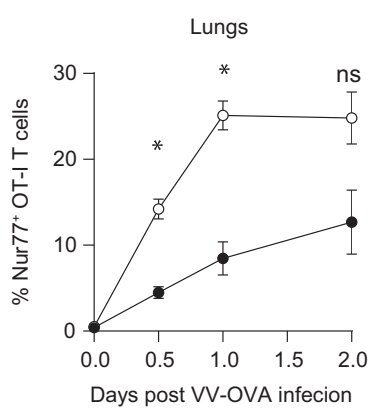

-o- Influenza (PR8-OVA)

- MCMV-ie2-SIINFEKL

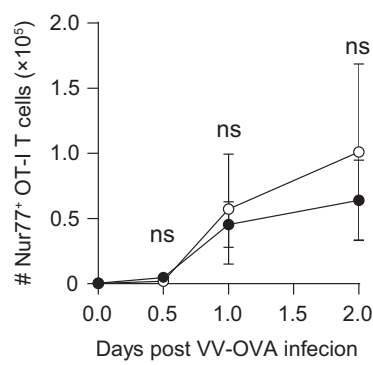

h

\section{.}

.

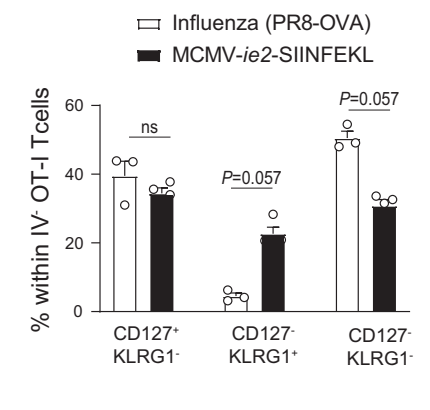

i

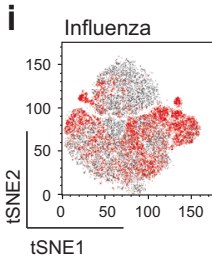

j

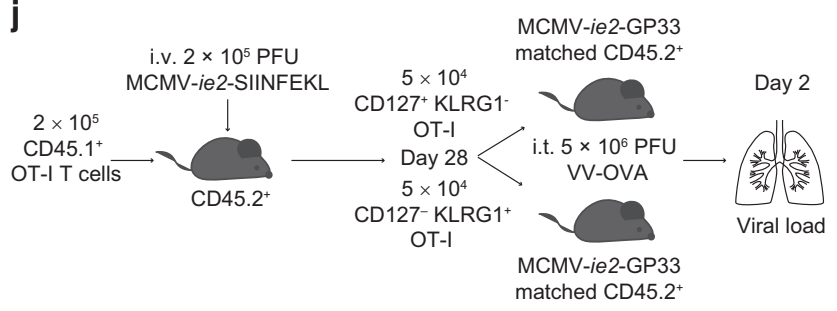

g
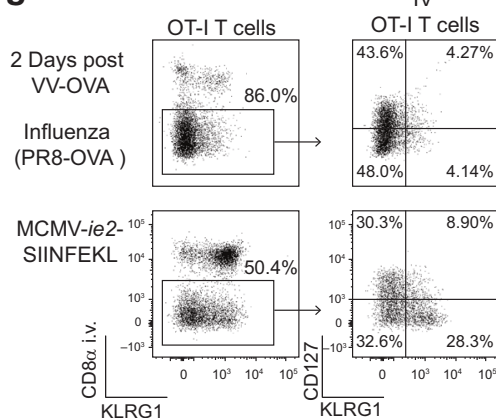

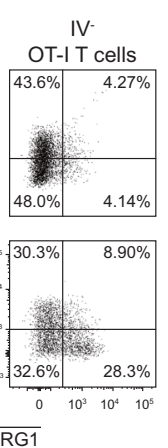

confirming the flow cytometry data (Fig. 5c). Also, influenza virus-induced memory $\mathrm{CD} 8 \mathrm{~T}_{\mathrm{RM}}$ cells relocated from their original location near Epcam ${ }^{+}$epithelial cells to more dispersed sites within the lung tissue.
To examine where in the lung tissue OT-I cells encountered antigen, we used OT-I T cells carrying a Nur77-green fluorescent protein (GFP) transgene and thereby reporting $T$ cell receptor (TCR) triggering ${ }^{42}$ to generate lung memory OT-I T cells. A larger 
Fig. 5 MCMV-induced memory OT-I T cells relocate in the lungs upon antigenic challenge. a Mice that either have memory OT-I T cells in the lungs induced by MCMV-ie2-SIINFEKL or influenza virus (PR8-OVA) (as described in Fig. 1a) were locally challenged on day 28 with $5 \times 10^{6}$ PFU VV-OVA. 2 days post-secondary challenge, the percentage of OT-I T cells that was in the vasculature was determined by i.v. injection of $5 \mu \mathrm{g}$ fluorescently conjugated $\alpha \mathrm{CD} 8$ antibody $3 \mathrm{~min}$ prior to euthanasia ( $n=14-19$, pooled from five independent experiments). $\mathbf{b}$ Mice that either had memory OT-I T cells in the lungs induced by MCMV-ie2-SIINFEKL or influenza virus (PR8-OVA) (as described in Fig. 1a) were locally challenged on day 28 with $3 \times 10^{5}$ PFU VV-G2 (a vaccinia virus that does not express the SIINFEKL epitope). 2 days post-secondary challenge, the percentage of OT-I T cells that was in the vasculature was determined by i.v. injection of $5 \mu \mathrm{g}$ fluorescently conjugated $\alpha \mathrm{CD} 8$ antibody 3 min prior to euthanasia $(n=12-14)$, pooled from four independent experiments). c Microscopy image shows the lung tissue 2 days postinfection with VV-OVA. d $5 \times 10^{4}$ CD45.1 ${ }^{+}$Nur77-GFP OT-I T cells were adoptively transferred into host mice that were subsequently i.t. infected with 50 PFU PR8-OVA or i.v. with $2 \times 10^{5}$ PFU MCMV-ie2-SIINFEKL. After 28 days, mice were i.t. challenged with $5 \times 10^{6}$ PFU VV-OVA. Flow cytometry plots show Nur77 expression on OT-I T cells in the lungs before rechallenge (left) and 2 days post VV-OVA infection (right). e The percentage (left) and total number (right) of OT-I T cells expressing GFP (Nur77) is shown in the lungs, after VV-OVA infection as mean \pm SEM ( $n=2-4$, one out of two experiments is shown). f Flow cytometry plots show in vivo CD8 $\alpha$ labeling and ex vivo CD8 $\beta$ staining on all OT-I $\mathrm{T}$ cells (left) or on GFP ${ }^{+}$OT-I T cells (right) one day post VV-OVA infection in the lungs. g Flow cytometry plots show in vivo CD8 $\alpha$ labeling versus KLRG1 expression on OT-I T cells, gated on CD45.1 $1^{+}$in the lungs, 2 days post VV-OVA infection (left), and CD127 KLRG1 staining on the IV $^{-}$fraction of the OT-I T cells (right). $\mathbf{h}$ Percentage of cells expressing CD127 and/or KLRG1 within the IV ${ }^{-}$fraction is shown ( $n=3-4$, one representative out of 3 independent experiments is shown). i tSNE clusters of CD45.1 $1^{+}$OT-I T cells in the lungs 2 days post VV-OVA infection, based on expression of Nur77, CD8 $\alpha$ i.v., CD127, KLRG1, CD44, CD69, and CD103. Expression of each marker is color-coded with red = expressed and black $=$ not expressed $\left(n=3\right.$ mice per group). $\mathbf{j}$ Experimental setup: $2 \times 10^{5}$ OT-I T cells were adoptively transferred into naive hosts that were subsequently infected with $2 \times 10^{5}$ PFU MCMV-ie2-SIINFEKL. After 4 weeks, CD127 ${ }^{+} \mathrm{KLRG}^{-}$and CD127 KLRG1 ${ }^{+}$were sorted from the spleen. $5 \times 10^{4}$ cells of each population were separately transferred into MCMV-ie2-GP33 infection-matched recipients, that were subsequently challenged with $5 \times 10^{6}$ PFU VV-OVA. Two days later the viral load was determined in the lungs. $\mathbf{k}$ The VV-OVA viral load in the lungs is shown, pooled data from two independent experiments is shown $(n=5-10)$. Geometric mean and limit of detection are indicated. I The total number of OT-I T cells in the lungs is shown $(n=10$, pooled from two independent experiments). All bar graphs show mean + SEM, each dot represents an individual mouse. Two-sided Mann-Whitney test (a and j) or two-way ANOVA followed by Sidak's multiple comparisons test (e) or Kruskal-Wallis test with Dunn's post hoc test (i) was used to determine statistical significance: ${ }^{*} P<0.05$, ${ }^{* * *} P<0.0001$ and not significant (ns) $P \geq 0.05$.

percentage of the influenza virus-induced memory $\mathrm{T}$ cells responded to antigen compared to MCMV-induced T cells, judged by a higher percentage of $\mathrm{GFP}^{+}$OT-I T cells at all time points of analysis (Fig. $5 d$, e). However, no differences were found in the total number of OT-I T cells responding to antigen (Fig. 5e). The Nur77-GFP signal reports TCR triggering but not inflammation, as no $\mathrm{GFP}^{+}$cells were detected upon antigenic challenge with VV that did not express OVA (Supplementary Fig. 5B). OT-I activation first occurred in the lungs, subsequently in the mediastinal LN (medLN) after $24 \mathrm{~h}$, but was not observed in the spleen in the first 2 days (Supplementary Fig. $5 \mathrm{C}$ ). In the lungs, all Nur $77^{+}$OT-I T cells were located in the $\mathrm{IV}^{-}$fraction, indicating that antigen encounter took place within the lung tissue and not in the vasculature (Fig. 5f).

$\mathrm{T}_{\mathrm{CM}}$ cells $\left(\mathrm{CD} 127^{+} \mathrm{KLRG}^{-} \mathrm{CD}^{-} \mathrm{L}^{+}\right)$were reported to traffic into inflamed tissue due to extensive synthesis of core $2 \mathrm{O}$-glycans that generate functional ligands for E- and P-selectins. ${ }^{43}$ Furthermore, $\mathrm{T}_{\mathrm{EM}}$ cells (CD127 ${ }^{+} \mathrm{KLRG}^{-} \mathrm{CD}^{-} 2 \mathrm{~L}^{-}$) express more core 2 O-glycans than the more terminally differentiated effector-like CD127 $\mathrm{KLRG}^{+} \mathrm{CD} \mathrm{LL}^{-} \mathrm{T}$ cells. As the MCMV-induced T cell pool consists of approximately $80 \%$ of $\mathrm{KLRG}^{+}$cells (Fig. 1c), we next determined which cells have the ability to migrate out of the vasculature. In the $\mathrm{IV}^{-}$fraction of the MCMV-induced OT-I T cell pool, we detected comparable percentages of $\mathrm{CD}_{12} 7^{+} \mathrm{KLRG}^{-}$, $\mathrm{CD}_{127^{-}} \mathrm{KLRG}^{+}$and $\mathrm{CD} 127^{-} \mathrm{KLRG}^{-}$cells, whereas in the influenza virus setting, hardly any $\mathrm{CD} 127^{-} \mathrm{KLRG}^{+}$cells were detected (Fig. $5 \mathrm{~g}, \mathrm{~h}$ ). These results confirm that $\mathrm{CD} 127^{+} \mathrm{KLRG}^{-}$ cells are superior in trafficking into inflamed tissues compared to $\mathrm{CD}_{127^{-}} \mathrm{KLRG}^{+}{ }^{+}$, as the $\mathrm{CD} 127^{-} \mathrm{KLRG}^{+}$subset is the dominant subset in the vasculature (Supplementary Fig. 5D). CD127 $\mathrm{KLRG}^{-}$OT-I cells, perhaps early stage effector cells, were also abundantly found in the $\mathrm{IV}^{-}$fraction in both the MCMV and influenza virus setting. Thus, in MCMV-primed mice, not only the most abundant $\mathrm{CD} 127^{-} \mathrm{KLRG}^{+}$OT-I T cell population migrated out of the vasculature into the lung tissue, but also some smaller populations. tSNE analysis revealed that antigen recognition occurred in the $\mathrm{IV}^{-}$fraction and that in the MCMV setting both $\mathrm{CD}_{12} 7^{+} \mathrm{KLRG}^{-}$and $\mathrm{CD} 127^{-} \mathrm{KLRG}^{+}$OT-I T cells responded to antigen (Fig. 5i). The Nur77 signal completely overlapped with CD69 expression. This is not surprising, as CD69 is an early activation marker for T cells upon TCR triggering. Some
Nur77 signal overlapped with $\mathrm{CD} 69^{+} \mathrm{CD} 103^{+}$cells, indicating that also $T_{R M}$ cells were responding to antigen. Interestingly, there was not such a clear separation for the MCMV- and influenza virusinduced T cells as observed in Fig. 1h, implying that the OT-I cells found in the lungs resemble each other more (Fig. 5i).

To test whether both $\mathrm{CD} 127^{+} \mathrm{KLRG}^{-}$and $\mathrm{CD} 127^{-} \mathrm{KLRG}^{+}$OT-I cells contributed to protection from a VV-OVA challenge, we sorted these subsets from MCMV-ie2-SIINFEKL-infected mice. Equal cell numbers were separately transferred into infectionmatched recipients that were infected with MCMV-ie2-GP33 not expressing the $\mathrm{OVA}_{257-264}$ epitope, to achieve a similar proinflammatory environment without an endogenous $\mathrm{OVA}_{257-264} /$ $\mathrm{K}^{\mathrm{b}}$-specific $\mathrm{T}$ cell response (Fig. 5j). Mice that received either $\mathrm{CD} 127^{+} \mathrm{KLRG}^{-}$or $\mathrm{CD} 127^{-} \mathrm{KLRG} 1^{+}$OT-I cells similarly controlled a local VV-OVA challenge (Fig. 5k). Furthermore, no differences were found in the total number OT-I T cells recovered from the lungs (Fig. 5l). These data indicate that when present in equal numbers, both $\mathrm{CD}_{12} 7^{+} \mathrm{KLRG}^{-}$and $\mathrm{CD} 127^{-} \mathrm{KLRG}^{+}$provide protection from a local VV challenge.

Hematopoietic cells in the lungs are infected by VV-OVA We next addressed which cells in the lungs are infected by $\mathrm{V}$ OVA. For this purpose, we generated a VV-OVA virus that also expresses GFP. Upon i.t. administration of this virus, the majority of the GFP signal was detected in $\mathrm{CD} 45^{+}$hematopoietic cells (Fig. 6a, b), mostly in $C^{2} 11 b^{+}$cells (Fig. 6c). VV-OVA-GFP-infected cells were also found in the lung parenchyma, corresponding to the location where OT-I T cells were observed upon VV-OVA infection (Figs. 5a and 6d).

Leukocytes can be attracted to inflammatory sites by chemokines which, together with integrin-mediated binding, facilitate the migration through the endothelium to the site of infection. ${ }^{44} \mathrm{~A}$ number of chemokines including CCL2, CCL3, CCL4, CCL5, CXCL9, and $C X C L 10$, were detected in lung homogenates upon VV-OVA infection. In mice that experienced an infection before the VV-OVA challenge, the chemokine concentrations were consistently higher as compared to VV-OVA infected naive mice (Fig. 6e). Correspondingly, the number of CD8 T cells (excluding the CD45.1 ${ }^{+}$OT-I $\mathrm{T}$ cells) in the $\mathrm{IV}^{-}$fraction was larger in memory compared to naive mice (Supplementary Fig. 6). No marked differences were found in the chemokine induction between MCMV- or influenza 
a

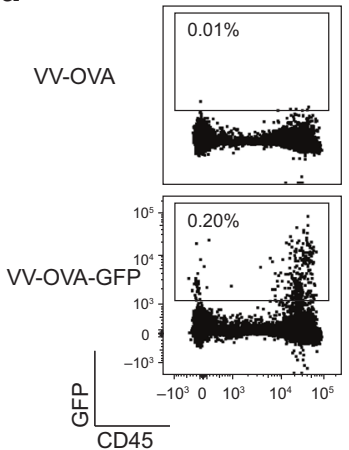

b

Lungs, 1 day post VV-OVA-GFP

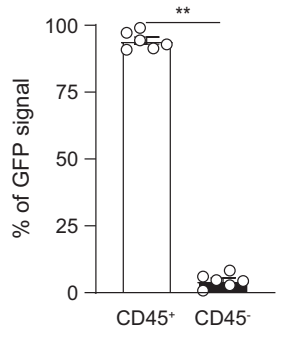

C

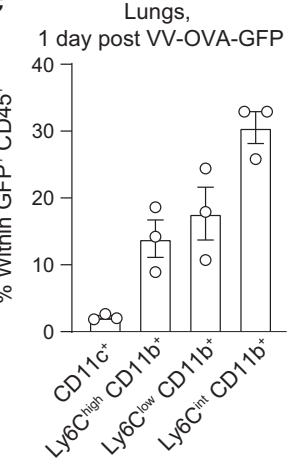

d Lungs,
1 day post W-OVA-GFP Epcam VV-OVA-GFP DAPI

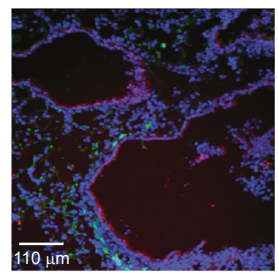

e
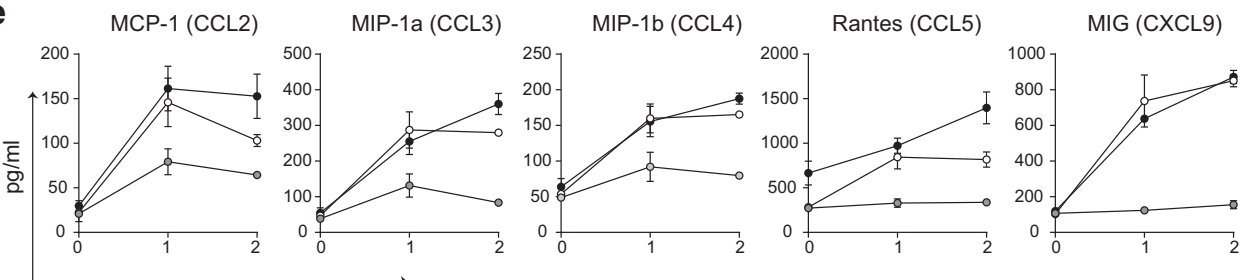

IP-10 (CXCL10)

Days post VV-OVA infection

- Naive

-o- Influenza (PR8-OVA) • MCMV-ie2-SIINFEKL

f

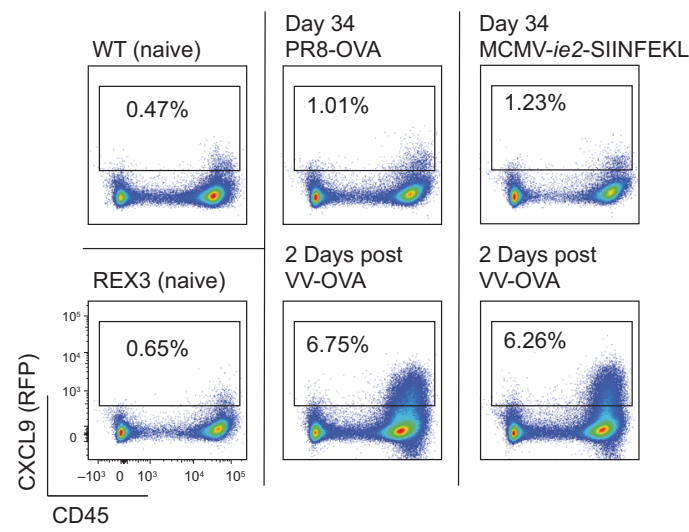

g
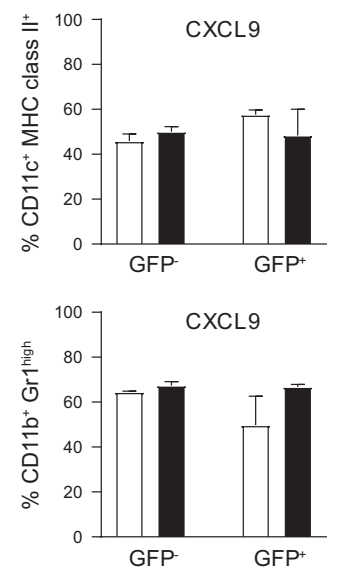

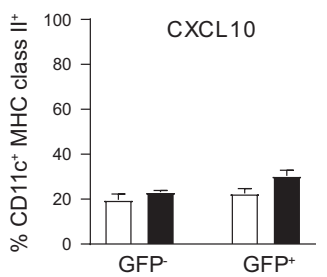

CXCL10

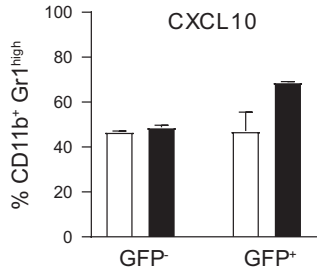

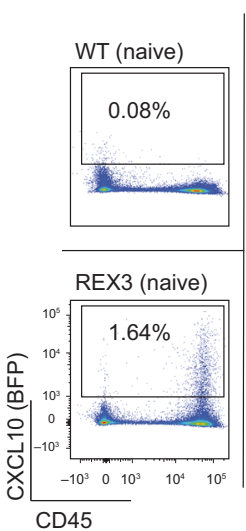

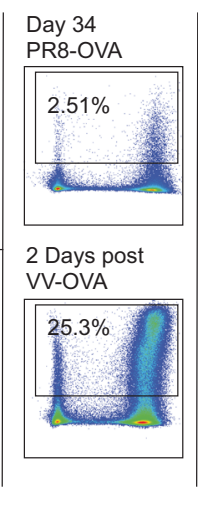

Day 34 MCMV-ie2-SIINFEKL

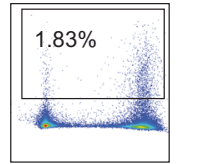

2 Days post

VV-OVA

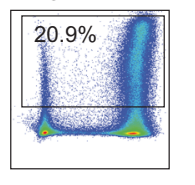

h

Lungs, 2 days post W-OVA infection

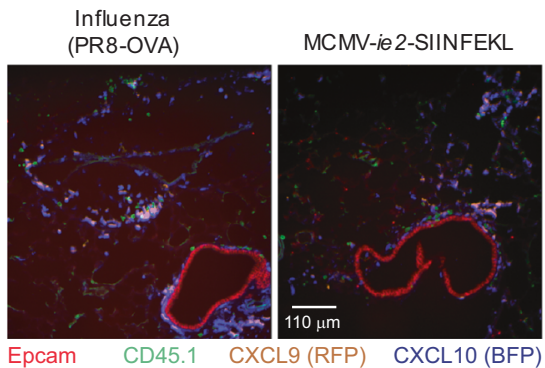

$\square$ Influenza (PR8-OVA)

- MCMV-ie2-SIINFEKL

Fig. 6 Hematopoietic cells in the lungs are infected by VV-OVA. Mice were infected with $5 \times 10^{6}$ PFU VV-OVA or VV-OVA-GFP. $24 \mathrm{~h}$ postinfection, the lungs were isolated. a Flow cytometry plots show GFP expression versus CD45. b Bar graph shows percentage of GFP signal in the hematopoietic $\left(C D 45^{+}\right)$and non-hematopoietic $\left(C D 45^{-}\right)$compartment as mean $+\mathrm{SEM}(n=6$, pooled from two independent experiments). Two-sided Mann-Whitney test was used to determine statistical significance: ${ }^{*} P<0.01$. c The distribution of the GFP-signal amongst the $\mathrm{CD}_{4} 5^{+}$compartment is shown as mean + SEM $(n=3$, one out of two independent experiments is shown). $\mathbf{d}$ Microscopy image shows the lungs 1 day post infection with VV-OVA-GFP. e Concentration of different chemokines in lung homogenates is shown as mean \pm SEM upon i.t. challenge with VV-OVA ( $n=3$, one out of two experiments is shown) in naive, influenza virus- and MCMV-infected mice. Day 0 represent day 28 post primary infection. f Flow cytometry plots show CXCL9 (RFP) and CXCL10 (BFP) production versus CD45 in REX3 mice. A WT and a naive REX3 mouse are included to determine the background level. $\mathbf{g}$ Bar graphs show the percentage of CXCL9 and CXCL10 that is produced by $\mathrm{CD} 11 \mathrm{c}^{+} \mathrm{MHC}$ class $\mathrm{II}^{+}$and $\mathrm{CD} 11 \mathrm{~b}^{+} \mathrm{Gr} 1^{\text {high }}$ cells in non-infected (GFP ${ }^{-}$) and $\mathrm{V}$-infected $\left(\mathrm{GFP}^{+}\right.$) cells in the lungs 2 days postinfection with $5 \times 10^{6}$ PFU VV-OVA-GFP. h Microscopy image shows the lungs of REX3 mice 2 days post infection with $5 \times 10^{6}$ PFU VV-OVA. 
virus-experienced mice. We confirmed the production of CXCL9 and CXCL10 upon VV-OVA infection using REX3 reporter mice that express red fluorescent protein (RFP) and blue fluorescent protein (BFP) under the control of the CXCL9 and CXCL10 promoter and found that hematopoietic cells, specifically CD11c $\mathrm{CHC}^{+}$class $\mathrm{II}^{+}$ and $\mathrm{CD} 11 \mathrm{~b}^{+} \mathrm{Gr} 1^{\text {high }}$ cells, were the main producers of both chemokines in both $\mathrm{V}$-infected and non-infected cells (Fig. 6f, g). CXCL9-producing and CXCL10-producing cells were localized in the lung parenchyma and lining the Epcam ${ }^{+}$airways (Fig. 6h). CD45.1 $1^{+}$OT-I T cells co-localized to these CXCL9 ${ }^{+}$and CXCL10 $0^{+}$ producing cells (Fig. $6 \mathrm{~h}$ ). Combined, these results show that upon a respiratory VV-OVA infection, hematopoietic cells are the main infected cells and that both infected and non-infected hematopoietic cells produce chemokines.

Chemokines are in part responsible for the extravasation of memory $T$ cells into the lung tissue

CXCL9 and CXCL10 bind to the chemokine receptor CXCR3. Although there was no difference in the induction of CXCL9 and CXCL10 in MCMV- and influenza virus-experienced mice, CXCR3 was differentially expressed on OT-I T cells. The majority of lunglocalized influenza virus-induced memory OT-I T cells expressed high levels of CXCR3, whereas CXCR3 was hardly detected on MCMV-induced OT-I T cells (Fig. 7a, b). CXCR3 expression was, however, detected on a subset of MCMV-induced OT-I T cells in the spleen (Supplementary Fig. 7A) and correlated with the expression of CD127 (Supplementary Fig. 7B). Upon VV challenge, OT-I T cells quickly downregulated CXCR3 (Fig. 7a, b). Although hardly any CXCR3-expressing cells were found directly ex vivo in the MCMV-setting, overnight incubation at $37^{\circ} \mathrm{C}$ of lung single cell suspensions revealed a proportion of cells expressing CXCR3, indicating that CXCR3 is actively downregulated within the lungs (Fig. 7c and Supplementary Fig. 7C). CXCR3 upregulation was hardly observed on OT-I T cells obtained from the spleen after overnight incubation (Supplementary Fig. 7D). Next, we determined the importance of CXCR3 signaling on T cell recruitment and positioning in the lungs (Fig. 7d). Blockade of CXCR3 during the VV-OVA challenge diminished the number of OT-I T cells that was found in the lung after VV-OVA challenge in MCMVexperienced mice (Fig. 7e), and the fraction of OT-I T cells associated with the vasculature was increased (Fig. 7f). A similar trend was observed in the influenza virus setting (Fig. 7e, f). In both settings, OT-I T cells were still found in the lung parenchyma and the positioning of these cells was not altered (Fig. 7g). Furthermore, no differences in the viral load were observed upon CXCR3 blockade (Supplementary Fig. 7E). These data show that CXCR3 signaling is in part involved in recruitment of memory OT-I $T$ cells to the lungs and from the vasculature into the lung parenchyma. However, in the absence of CXCR3 signaling, there are still sufficient numbers of antigen-specific cells present in, or recruited into the lung parenchyma to control VV-OVA replication.

Signaling via chemokines occurs via G-protein-coupled receptors, and this signaling is inhibited by pertussis toxin (PTX). As we observed a large range of different chemokines produced upon VV-OVA challenge, mice were administrated with PTX during the VV-OVA challenge period to inhibit all chemokine receptor signaling (Fig. 7h). PTX treatment partially inhibited the migration of MCMV-induced OT-I T cells from the vasculature into the lung tissue (Fig. 7i, j). However, no difference in the viral load was observed upon PTX treatment (Supplementary Fig. 7F). Similarly to CXCR3 blockade, the repositioning of the OT-I T cells to the lung parenchyma remained normal in both the MCMV- and the influenza virus-setting (Fig. 7k). These data indicate that chemokines are, at least in part, responsible for the recruitment and relocation of memory OT-I T cells from the vasculature deeper into the lung tissue, but other mechanisms also operate to control VVOVA replication.

\section{DISCUSSION}

Memory CD8 T cells residing in peripheral tissues are of interest in the context of vaccination. The memory $T$ cell subset providing the best protection in a given infection may vary. By using distinct viral infections, we induced memory $T$ cell pools with unique characteristics within the lung tissue and compared their protective capacity towards a local VV challenge. Upon influenza virus infection, the majority of the established memory $T$ cells had a $T_{R M}$ phenotype, whereas lung localized MCMV-specific CD8 $T$ cells predominantly expressed KLRG1 and thus exhibited an effector-like phenotype. Factors that contribute to the induction of a specific phenotype include the route and dose of infection, the viral tropism and the pro-inflammatory environment. For instance, only respiratory and not systemic administration of MCMV or influenza virus results in the formation of lung $T_{R M}$ cells. ${ }^{34,35,45}$ Moreover, antigen is required for the maintenance of the MCMVspecific inflationary $T$ cell pool and the MCMV inoculum dose impacts on the balance between $\mathrm{CD} 127^{+}$and $\mathrm{KLRG} 1^{+} \mathrm{MCMV}$ specific CD8 T cells found within the lung tissue. ${ }^{27,46,47}$ Differences in the localization between MCMV- and influenza virus-induced memory T cells were also observed. CD103 tethers $T_{\text {RM }}$ cells to Ecadherin expressed by epithelial cells, likely explaining their localization in close proximity to epithelial structures. MCMVspecific KLRG $1^{+}$cells express CX3CR $1,{ }^{48}$ which could explain their localization to the vasculature.

Despite their differences in anatomical localization, MCMVand influenza virus-induced memory $\mathrm{T}$ cells both provided protection from a local respiratory $\mathrm{VV}$ infection. We detected $\mathrm{V}$ mostly in hematopoietic cells. It is plausible that upon infection with other pathogens, a different outcome in protective capacity may be observed. For instance, intra-nasal administration of MCMV vectors encoding influenza virus epitopes facilitates the clearance of influenza virus in a $\mathrm{T}_{\mathrm{RM}}$-dependent manner. ${ }^{35}$ Despite having large number of effector-like cells in the lungs, mice without $T_{R M}$ cells had a higher viral load in the lungs, indicating that in an influenza virus setting, $\mathrm{T}_{\mathrm{RM}}$ cells are superior in protection as compared to circulating memory $T$ cells. Furthermore, $T_{\mathrm{RM}}$ cells induced by intra-nasal administration of MCMV-vectors encoding respiratory syncytial virus (RSV) epitopes also enhanced the protection towards RSV infection, compared to a setting in which only circulatory memory $T$ cells were present. ${ }^{34}$ As both influenza virus and RSV infect alveolar epithelial cells $s^{49-51}$ and $T_{R M}$ cells are lining the epithelial walls, their anatomical localization could give them an advantage over vasculature-associated memory CD8 T cells induced by systemic administration of MCMV.

To control VV replication, effector cytokines such as IFN- $\gamma$ and TNF are critical. ${ }^{38,39}$ Although we observed a decrease in the number of lung resident memory CD8 T cells 60 days postinfluenza virus infection, the remaining population was still protective. It could be that very few memory T cells are actually needed to provide protection in our experimental setup. In Fig. 5k, only 500 cells were recovered from the lungs after VV-OVA challenge, and a slight, but significant protective effect was observed compared to mice without any memory OT-I T cells. It has been shown before that the number of VV-specific memory CD8 T cells in the lung corresponds to their protective capacity. ${ }^{52}$ Of note, the number of cells responding to secondary VV-OVA antigen exposure, quantified by Nur77-GFP expression, did not differ between influenza virus- and MCMV-immune mice (Fig. 5e). IFN- $\gamma$ can be sensed up to a distance of $800 \mu \mathrm{m}$ from its producer in tumor tissue, ${ }^{53}$ indicating that IFN- $\gamma$ can act beyond the cells that are in direct contact with $\mathrm{T}$ cells. Whether this range is achieved in case of respiratory $\mathrm{VV}$ infections remains to be determined. In any case, we readily detected the IFN- $\gamma$-induced chemokines CXCL9 and CXCL10 throughout the lung tissue in REX3 mice. 


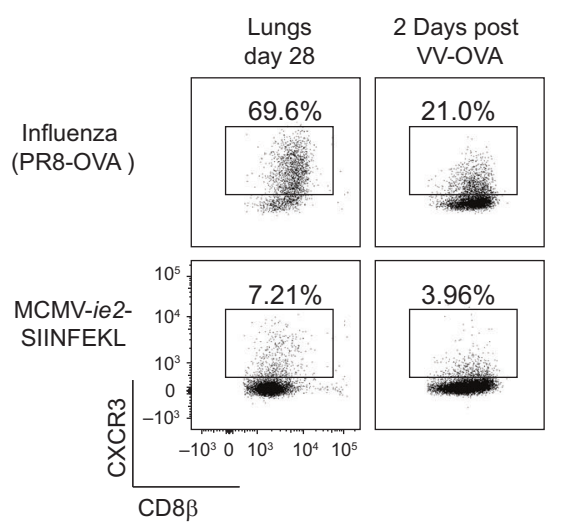

b

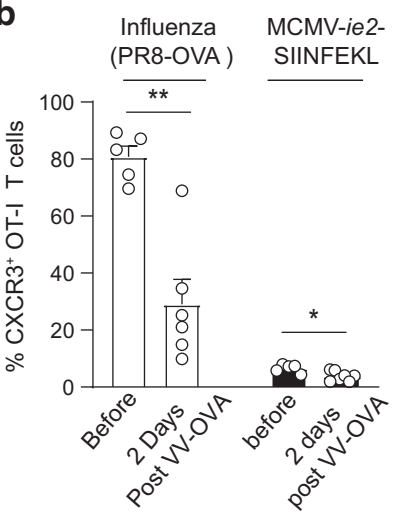

C

Lungs, OT-I T cells

- O/N $37^{\circ} \mathrm{C}$

Directly ex vivo

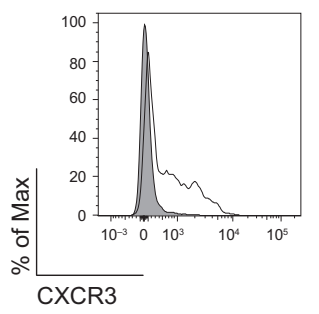

d

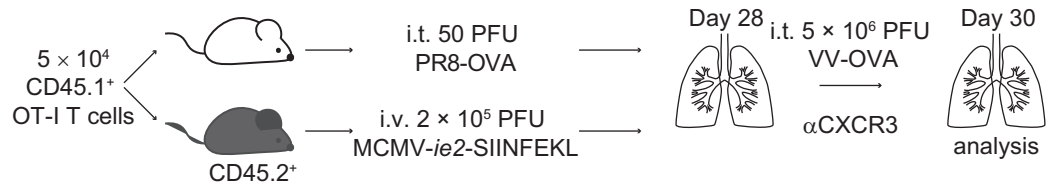

e $\begin{array}{cc}\text { Influenza } & \text { MCMV-ie2- } \\ \text { (PR8-OVA) } & \text { SIINFEKL }\end{array}$

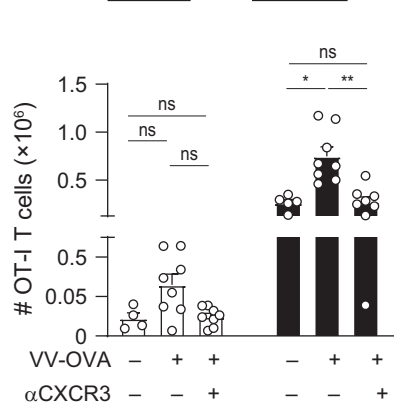

$\mathbf{f}$ (PR8-OVA) SIINFEKL

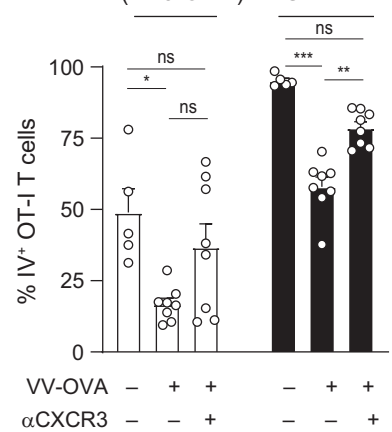

g

$$
\begin{gathered}
\text { Influenza } \\
\text { (PR8-OVA) }
\end{gathered}
$$

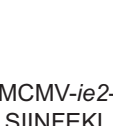

SIINFEKL
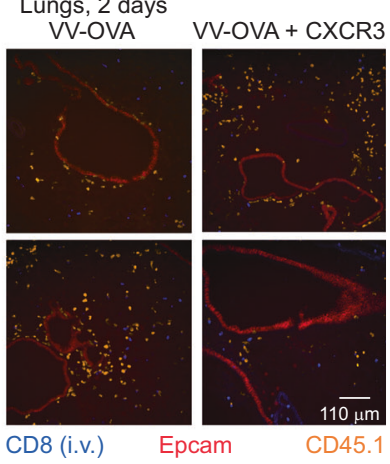

h
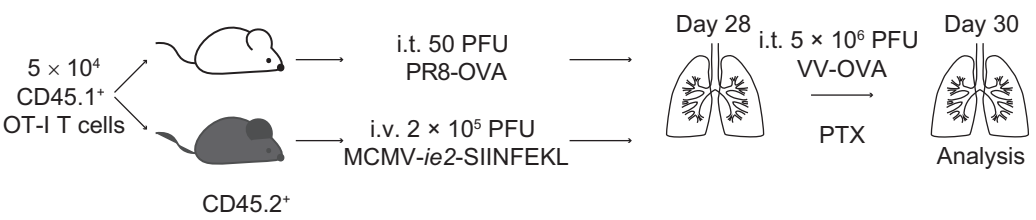

i

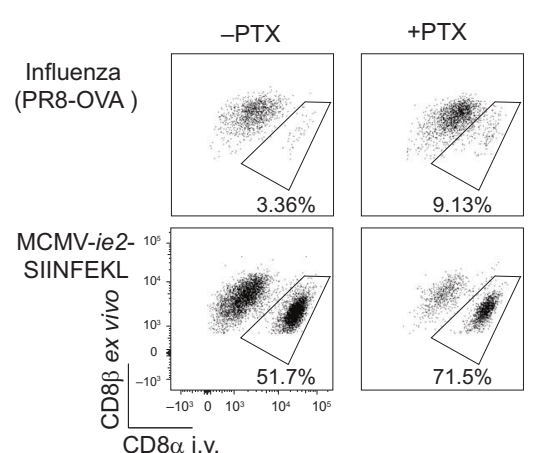

j

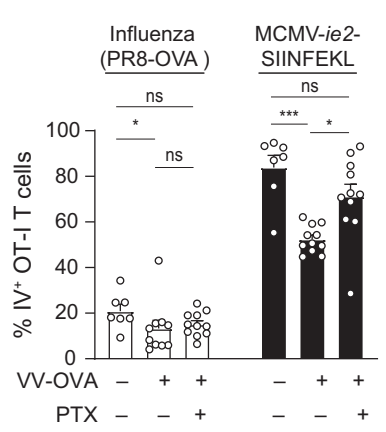

k

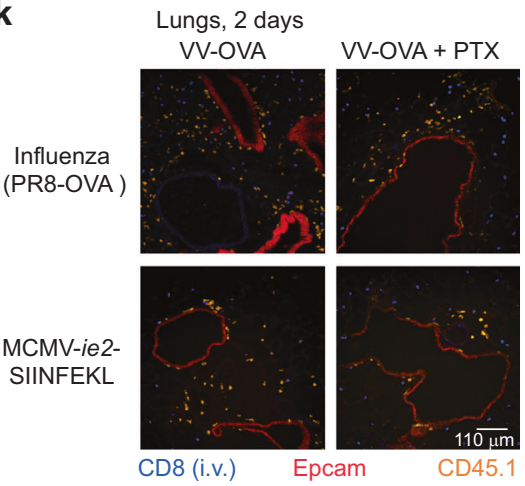

We found that upon local inflammation, MCMV-induced T cells extravasate in an antigen-independent manner and chemokines played a partial role in this process. Integrin-mediated extravasation likely contributes as well. ${ }^{44}$ In the MCMV setting, the increase in the lung localized OT-I T cell pool was mostly due to T cell recruitment, whereas in the influenza setting both $T$ cell recruitment and proliferation likely contributed. In the female reproductive tract, $\mathrm{T}_{\mathrm{RM}}$ cell activation recruits other cells of the immune system into the tissue, including bystander memory T cells, via IFN- $\gamma$-mediated upregulation of VCAM- 1 and by local induction of CXCL9 and CXCL10. ${ }^{14,54}$ Also in the lungs, $T_{R M}$ cell activation facilitates the accumulation of memory CD8 T cells upon influenza virus infection. ${ }^{35}$ In our experimental setup, migration of antigen-specific memory $T$ cells from the vasculature 
Fig. 7 Chemokines are partially involved in the extravasation of memory $\mathrm{T}$ cells into the lungs. a Flow cytometry plots show expression of CXCR3 on OT-I T cells isolated from the lungs, 28 days post primary infection (left) or 2 days post rechallenge with VV-OVA (right). $\mathbf{b}$ Bar graphs show percentage of OT-I T cells expressing CXCR3 in the lungs, as mean + SEM $(n=5-6$, pooled from two independent experiments). Each dot represents an individual mouse. c Histogram shows CXCR3 expression of OT-I T cells in the lungs either directly ex vivo or after overnight incubation at $37^{\circ} \mathrm{C}$. Cells are isolated from an MCMV experienced mouse 2 days post infection with $5 \times 10^{6}$ PFU VV-OVA. d Experimental setup: $5 \times 10^{4}$ CD45.1 ${ }^{+}$OT-I T cells were adoptively transferred into CD45.2 $2^{+}$hosts that were either infected i.t. with 50 PFU PR8-OVA or i.v. with $2 \times$ $10^{5}$ PFU MCMV-ie2-SIINFEKL. Twenty-eight days post-infection, mice were i.t. challenged with $5 \times 10^{6}$ PFU VV-OVA. Mice received $250 \mu g$ $\alpha C X C R 3$ blocking antibodies one day prior and during the rechallenge period. e Bar graphs show the total number of OT-I T cells recovered from the lungs as mean + SEM ( $n=4-8$, pooled from two independent experiments). $f$ Bar graphs show the percentage of OT-I T cells associated with the vasculature determined by i.v. injection of $5 \mu \mathrm{g}$ fluorescently conjugated $\alpha \mathrm{CD} 8$ antibody 3 min prior to euthanasia $(n=$ 4-8), pooled from two independent experiments). g Microscopy images show the lung 2 days post infection with $5 \times 10^{6}$ PFU VV-OVA. h Experimental setup: $5 \times 10^{4}$ CD45.1 $1^{+}$OT-I T cells were adoptively transferred into CD45.2 ${ }^{+}$hosts that were either infected i.t. with 50 PFU PR8-OVA or i.v. with $2 \times 10^{5}$ PFU MCMV-ie2-SIINFEKL. Twenty-eight days post-infection, mice were i.t. challenged with $5 \times 10^{6}$ PFU VV-OVA. Mice received $400 \mathrm{ng}$ PTX i.p. on the day of and one day after VV-OVA rechallenge. i Flow cytometry plot shows in vivo CD8 $\alpha$ labeling and ex vivo CD8 $\beta$ staining on OT-I T cells in the lungs 2 days post VV-OVA infection upon injection with $5 \mu \mathrm{g}$ fluorescently conjugated $\alpha C D 8$ antibody 3 min prior to euthanasia. j Bar graphs shows the percentage of IV ${ }^{+}$OT-I T cells in the lungs 2 days post VV-OVA infection ( $n=7-11$, pooled from three independent experiments) as mean + SEM. k Microscopy images show the lung 2 days post infection with $5 \times 10^{6}$ PFU VVOVA. Two-sided Mann-Whitney test $\mathbf{b}$ or Kruskal-Wallis test with Dunn's post hoc $(\mathbf{e}, \mathbf{f}, \mathbf{j})$ test was used to determine statistical significance: ${ }^{*} P<0.05,{ }^{* *} P<0.01,{ }^{* * *} P<0.001$ and not significant (ns) $P \geq 0.05$.

into the lung parenchyma was more pronounced in the MCMV as compared to the influenza virus setting, likely due to the large numbers of memory cells that are present in the circulation upon MCMV infection. We observed a similar recruitment of bystander CD8 T cells into lung parenchyma in both the MCMV and influenza virus setting (Supplementary Fig. 6). Lung-localized MCMVinduced T cells were potent IFN- $\gamma$ producers, and both CXCL9 and CXCL10 were produced upon VV-OVA infection. Recently, it was shown that $\mathrm{T}_{\mathrm{RM}}$ cells in the female reproductive tract and the skin maintain their proliferative capacity. ${ }^{40,41}$ Here we observed local proliferation of memory $T$ cells in the lungs upon antigen exposure in the influenza virus setting. However, whether the $T_{R M}$ cells or other memory $T$ cell subsets were proliferating remains to be determined.

Upon lung inflammation, both MCMV-specific $\mathrm{KLRG}^{+}$and CD $127^{+}$OT-I T cells extravasated from the vasculature. CD $127^{+}$ cells were superior in entering the lung tissue as a higher percentage of $\mathrm{CD} 127^{+}$cells was found within the lung tissue as compared to the blood. This is likely due to higher Core 2 Oglycan synthesis in $\mathrm{CD} 127^{+}$cells that enables these cells to traffic into inflamed tissue. ${ }^{43}$ Both $\mathrm{CD} 127^{+}$and $\mathrm{KLRG} 1^{+}$cells were able to control VV-infection in the lungs. We have shown before that the number of $\mathrm{KLRG}^{+}$antigen-specific cells in the ovaries correlated with the degree of protection towards an i.p. VV challenge. ${ }^{2}$ These differences might be explained by tissue-specific factors, as the lung is highly vascularized. Furthermore, the ovaries are the preferred site of VV replication and therefore VV might infect also non-hematopoietic cells in this organ.

In many infection and vaccination settings, CXCR3 expression is required for $T$ cells to enter the lung tissue. ${ }^{55,56}$ Lung entry subsequently affects CXCR3 expression in an antigen-dependent manner ${ }^{57}$ and CXCR3 $3^{\text {low }}$ cells are preferentially found in close proximity to the vasculature. ${ }^{57}$ However, both CXCR3 ${ }^{\text {high }}$ and CXCR3 $3^{\text {low }}$ cells can provide protection from respiratory infections. $^{56-59}$ The signals required for the downregulation of CXCR3 are not entirely clear. We found that MCMV-induced memory $T$ cells in the lung do not express CXCR3 ex vivo, but upon overnight incubation its expression was restored, likely indicating that the lung environment actively promotes CXCR3 downregulation. Blocking of $\mathrm{CXCR} 3$ during the rechallenge period diminished the number of cells recovered from the lung, indicating that CXCR3 is at least in part involved in the recruitment of memory OT-I cells to the lung. In the MCMV setting, CXCR3 is mostly expressed by $\mathrm{CD} 127^{+}$cells (Supplementary Fig. 7B), potentially recruiting these cells upon VV-OVA challenge.

MCMV-induced memory T cells are stably maintained over time, making CMV-vectors an interesting candidate for $\mathrm{T}$ cell-based vaccines. Until day 60 post-infection, we did not observe a decrease in the protective capacity of influenza virus-induced memory $T$ cells, despite a reduction in $T_{R M}$ cells. It is conceivable that a loss in protective capacity would become evident at later time points after influenza virus infection. Here we set out to determine which cells are superior in providing early protection from a local viral challenge. This likely depends on the timing of secondary infection as well as on the local tropism of the recall pathogen. For future vaccine approaches aimed at eliciting $T$ cells at mucosal sites, it is important to consider which memory $T$ cell subset provides protection from a specific pathogen and therefore should be induced by the vaccines. Our data show that in case of a respiratory $\mathrm{VV}$ infection, both $\mathrm{T}_{\mathrm{RM}}$ cells and MCMV-induced circulatory cells fulfill this requirement.

\section{METHODS}

Ethics statement

This study was conducted in accordance to the guidelines of the animal experimentation law (SR 455.163; TVV) of the Swiss Federal Government. The protocol was approved by Cantonal Veterinary Office of the canton Zürich, Switzerland (Permit number 168/2015, $114 / 2017$ and 58/2020).

\section{Mice}

C57BL/6J were purchased from Janvier Elevage and were used as WT mice. The congenic CD45.1 (Ly5.1) and the C57BL/6-Tg (TcraTcrb) $1100 \mathrm{Mjb} / \mathrm{J}$ (OT-I) mice were bred in house. Nur77-GFP OT-I mice were generated by crossing the OT-I mouse with the Nur77-GFP reporter mouse. ${ }^{42}$ REX3 mice expressing RFP and BFP under the control of the CXCL9 and CXCL10 promoter were obtained from Prof. Dr. Matteo lannacone and are described. ${ }^{60}$ All mice were housed and bred under specific pathogen-free facilities at the Eidgenössische Technische Hochschule (ETH) Hönggerberg. All mice were between 7 and 12 weeks at the start of each experiment and were age- and sex-matched.

Viruses

The recombinant influenza $A$ virus (IAV) strain A/PR8 expressing $\mathrm{OVA}_{257-264}{ }^{61}$ was a kind gift from Stephen Turner (St. Jude Children's Research Hospital, Memphis, USA) and was grown for 2 days at $35^{\circ} \mathrm{C}$ in the allantoic cavities of $10-11$-day-old fertile chicken eggs. Viral titers were quantified by standard plaque assay using Madin-Darby canine kidney cells. Mice were infected with 50 PFU intra tracheally (i.t.) in $50 \mu \mathrm{l}$ PBS. For an i.t. infection mice were anesthesized by isoflurane inhalation or by an i.p. injection with $5 \mu \mathrm{g}$ xylazine $/ 100 \mu \mathrm{g}$ ketamine per gram of body weight.

MCMV-ie2-SIINFEKL and MCMV-ie2-GP33 are obtained from Dr. L. Cicin-Sain, contain the $\mathrm{m} 157$ gene and are described. 2,62,63 Mice 
were infected with $2 \times 10^{5}$ PFU either i.v. or i.t.. MCMV stocks were propagated on M2-10B4 cells. The virus was subsequently purified by ultracentrifugation using a $15 \%$ sucrose cushion. Viral titers were determined by standard plaque assay as described. ${ }^{64}$ Recombinant VV (Western Reserve) expressing Ovalbumin protein (VV-OVA) inserted into the thymidine kinase gene was grown on BSC40 cells and was provided by Dr. P. Klenerman (Oxford Univeristy). Recombinant VV expressing the LCMV glycoprotein (VV-G2) was originally obtained from Dr. D.H.L. Bishop (Oxford University) and was grown on BSC40 cells at low multiplicity of infection. Recombinant Western Reserve vaccinia Ovalbumin nuclear-eGFP virus (WR OVA NP-eGFP, VV-OVA-GFP) was generated by inserting pNP-SIINFEKL-eGFP into the A56L locus of Western Reserve vaccinia Ovalbumin virus (WR VV-OVA) using homologous recombination as described previously. ${ }^{65-67}$ Briefly, BSC40 cells were infected with VV-OVA WR, transfected with linearized plasmid $4 \mathrm{~h}$ post infection and harvested $48 \mathrm{~h}$ post infection. Clonal recombinant fluorescent virus was selected though multiple serial dilution infection rounds. Mice were challenged i.t. with $5 \times 10^{6}$ PFU VV (unless stated otherwise) in $50 \mu \mathrm{l}$ under anasthesia as described.

Plaque assays

To determine the viral load in the lungs of VV-infected mice, $1.5 \times$ $10^{5}$ BSC40 cells were plated per well of a 24-well plate in MEM supplemented with 5\% FCS, penicilin, streptamycin, and glutamin. The following day, lungs of V-infected mice were lysed two times for $1.5 \mathrm{~min}$ at $25 \mathrm{~Hz}$ using a tissue lyser (Qiagen). Samples were centrifuged for $5 \mathrm{~min}$ at $3.5 \times \mathrm{g}$ and supernatant was used. $200 \mu \mathrm{l}$ of serial dilutions of homogenized lung lysates were propagated on BSC40, upon which the plates were incubated for $1 \mathrm{~h}$ at $37^{\circ} \mathrm{C} .1 \mathrm{ml}$ of complete MEM medium was added and plates were incubated for another day at $37^{\circ} \mathrm{C}$. Plates were fixed for $20 \mathrm{~min}$ by adding $200 \mu \mathrm{l}$ of $4 \%$ formalin and subsequently developed using crystal violet solution.

\section{Flow cytometry}

Single cell suspensions were prepared from spleen by meshing the tissue through a $70 \mu \mathrm{M}$ cell strainer. Erythrocytes were lysed using a hypotonic ammonium-chloride-potassium buffer for 1 min. To prepare a single cell suspension from the lungs, mice were perfused with PBS to remove all blood-associated cells, the tissue was cut into small pieces, subsequently incubated with Collegenase I and DNAse I for $45 \mathrm{~min}$, followed by a $30 \%$ percoll gradient. To determine the lung tropism of VV-OVA-GFP or the production of CXCL9 and CXCL10, no percoll gradient was used. Cells were incubated with fluorescently conjugated antibodies for $30 \mathrm{~min}$ at $4{ }^{\circ} \mathrm{C}$. Dead cells were excluded using a LIVE/DEAD fixable NEAR-IR staining. For intracellular Ki67 staining, the FoxP3 kit (invitrogen) was used according to manufacterer's protocol. Multi-parametric flow cytometric analysis was performed using LSRII flow cytometer (BD Biosciences) or LSRFortessa (BD Biosciences) with FACSDiva software. Data was analyzed using FlowJo software (Tree Star). Cells were sorted using a FACS Aria sorter.

\section{Chemokine bead assay}

To determine the concentration of different chemokines in the lungs, mice were perfused with PBS, lungs were snap-frozen in liquid nitrogen and stored at $-80^{\circ} \mathrm{C}$ until further use. Lungs were homogenized in $1 \mathrm{ml}$ of MEM supplemented with $5 \%$ FCS, penicilin, streptamycin, and glutamin and were lysed two times for $1.5 \mathrm{~min}$ at $25 \mathrm{~Hz}$ using a tissue lyser (Qiagen). Samples were centrifuged for $5 \mathrm{~min}$ at $3.5 \times \mathrm{g}$ and supernatant was used. To determine the chemokine concentration the mouse proinflammatory chemokine panel (Biolegend) was used with vbottom plate according to manufacturers protocol. Data was analzed with the Legendplex analysis software (Biolegend).
MHC class I tetramers and in vitro restimulations

MHC class I monomers for $\mathrm{OVA}_{257-264}$ ( $\mathrm{K}^{\mathrm{b}}$ restricted) were produced as described ${ }^{68}$ and were tetramerized using streptavidin-APC. For in vitro restimulation, cells were stimulated for $5 \mathrm{~h}$ at $37^{\circ} \mathrm{C}$ with $1 \mu \mathrm{g} / \mathrm{ml} \mathrm{OVA}_{257-264}$ peptide in the presence of $2 \mu \mathrm{g} / \mathrm{ml}$ Brefeldin A. Cell surface staining was performed as described, and cells were subsequently fixed with $0.5 \%$ PFA overnight. The following day, intracellular cytokine staining was performed by diluting the antibodies in perm/wash buffer (Life Technologies) and incubating the samples for $30 \mathrm{~min}$ at $4{ }^{\circ} \mathrm{C}$.

\section{Adoptive transfer, FTY720, PTX, CXCR3 blockade and BrdU} treatment

OT-I or Nur77 OT-I CD8 T cells were enriched from spleens by negative selection according to manufacterer's protocol (Mojosort, biolegend). $5 \times 10^{4}$ TCR transgenic OT-I cells were adoptively transferred into new hosts that were subsequently infected with influenza virus or MCMV-expressing $\mathrm{OVA}_{257-264}$. For microscopy analysis, $1 \times 10^{5}$ OT-I T cells were transferred into naive recipients prior to infection. To inhibit $\mathrm{T}$ cell recruitment from secondary lymphoid organs, mice received $25 \mu \mathrm{g}$ FTY720 on day 0 and 1 of VV-OVA infection i.p. and starting at day 0, FTY720 was administrated in the drinking water at a concentration of $5 \mu \mathrm{g} / \mathrm{ml}$. To inhibit chemokine receptor signaling, mice received 400 ng PTX i.p. on the day and one day after VV-OVA challenge. For CXCR3 blockade, mice received $250 \mu \mathrm{g}$ aCXCR3 antibodies (clone CXCR3-173, BioXCell) i.p. on day $-1,0$, and 1 post VV-OVA challenge. To determine proliferation, mice received $0.8 \mathrm{mg} / \mathrm{ml}$ BrdU in the drinking water starting on the day of rechallenge. BrdU staining was performed according to manufacturer's protocol for BrdU flow kit (BD).

\section{Immunofluorescence microscopy}

To fix the lungs, $1 \mathrm{ml}$ of $1 \%$ PFA was infused into the lungs of euthanized mice with a cannula inserted via a small incision in the trachea. After 20 min, PFA was removed and was replaced by $1 \mathrm{ml}$ of $20 \%$ sucrose. After an additional incubation of $20 \mathrm{~min}$, sucrose was replaced by $1 \mathrm{ml}$ of optimum cutting temperature (OCT). The lungs were removed, frozen in liquid nitrogen and stored at $-20^{\circ} \mathrm{C}$ until further use. Cryosections of $10 \mu \mathrm{M}$ were made and air dried. For the staining, slides were re-hydrated in PBS and blocked with $10 \%$ rat serum in PBS. Slides were stained with antibodies diluted in PBS containing 1\% rat serum and mounted with Mowiol. Within 1 day, images were acquired on a Visitron confocal system inverse confocal microscope with $\times 10$ magnification. Data was analyzed using Volocity software.

\section{Statistical analysis}

Graphpad prism 8.0 software was used to calculate significance between samples. $P$-values $<0.05$ were considered as significant. Statistical test is indicated in each figure.

\section{ACKNOWLEDGEMENTS}

We thank N. Oetiker and F. Wagen for excellent technical assistance and members of the Oxenius, Joller, and Sallusto groups for helpful discussion. This work was supported by the ETH post-doctoral Fellowship program (FEL29 15-2 to S.P.M.W.), the Helmut Horten foundation (S.P.M.W.), by the SNF (IZHRZO_180552 to A.O.), the MRC program grant (MC_UU12018/7 to J.M.), and the European Research Council (649101UbiProPox to J.M.).

\section{AUTHOR CONTRIBUTIONS}

S.P.M.W. and A.O. conceived and designed the project and the experiments. S.P.M.W., J.O., V.Y., S.R.B., and V.G. performed experiments. S.P.M.W. analyzed the data. J.O., V.Y., S.R.B., V.G., J.M., and R.S. provided essential tools. S.P.M.W. writing original draft. A.O. and R.S. writing review and editing. A.O. supervised the study. S.P.M.W., J.M., and A.O. acquired funding. All authors reviewed the paper. 


\section{FUNDING}

Open Access funding provided by ETH Zurich.

\section{ADDITIONAL INFORMATION}

The online version of this article (https://doi.org/10.1038/s41385-020-00373-4) contains supplementary material, which is available to authorized users.

Competing interests: The authors declare no competing interests.

Publisher's note Springer Nature remains neutral with regard to jurisdictional claims in published maps and institutional affiliations.

\section{REFERENCES}

1. Martin, M. D. \& Badovinac, V. P. Defining memory CD8 T cell. Front. Immunol. 9, 2692 (2018).

2. Baumann, N. S. et al. Early primed KLRG1- CMV-specific T cells determine the size of the inflationary T cell pool. PLoS Pathog. 15, e1007785 (2019).

3. Borkner, L. et al. Immune protection by a cytomegalovirus vaccine vector expressing a single low-avidity epitope. J. Immunol. 199, 1737-1747 (2017).

4. Karrer, U. et al. Expansion of protective CD8+ T-cell responses driven by recombinant cytomegaloviruses. J. Virol. 78, 2255-2264 (2004).

5. Gerlach, C. et al. The chemokine receptor CX3CR1 defines three antigenexperienced CD8 T cell subsets with distinct roles in immune surveillance and homeostasis. Immunity 45, 1270-1284 (2016).

6. Jameson, S. C. \& Masopust, D. Understanding subset diversity in T cell memory. Immunity 48, 214-226 (2018).

7. Jiang, $X$. et al. Skin infection generates non-migratory memory CD8+ T(RM) cells providing global skin immunity. Nature 483, 227-231 (2012).

8. Sathaliyawala, T. et al. Distribution and compartmentalization of human circulating and tissue-resident memory T cell subsets. Immunity 38, 187-197 (2013).

9. Schenkel, J. M. \& Masopust, D. Tissue-resident memory T cells. Immunity 41, 886-897 (2014)

10. Smith, C. J., Caldeira-Dantas, S., Turula, H. \& Snyder, C. M. Murine CMV infection induces the continuous production of mucosal resident T cells. Cell Rep. 13, 1137-1148 (2015).

11. Thom, J. T., Weber, T. C., Walton, S. M., Torti, N. \& Oxenius, A. The salivary gland acts as a sink for tissue-resident memory $\mathrm{CD} 8(+) \mathrm{T}$ cells, facilitating protection from local cytomegalovirus infection. Cell Rep. 13, 1125-1136 (2015).

12. Wakim, L. M., Gebhardt, T., Heath, W. R. \& Carbone, F. R. Cutting edge: local recall responses by memory $T$ cells newly recruited to peripheral nonlymphoid tissues. J. Immunol. 181, 5837-5841 (2008).

13. Welten, S. P. M., Sandu, I., Baumann, N. S. \& Oxenius, A. Memory CD8 T cell inflation vs tissue-resident memory $T$ cells: same patrollers, same controllers? Immunol. Rev. 283, 161-175 (2018).

14. Schenkel, J. M. et al. T cell memory. Resident memory CD8 T cells trigger protective innate and adaptive immune responses. Science 346, 98-101 (2014)

15. Ariotti, S. et al. T cell memory. Skin-resident memory CD8(+) T cells trigger a state of tissue-wide pathogen alert. Science 346, 101-105 (2014).

16. O'Hara, G. A., Welten, S. P., Klenerman, P. \& Arens, R. Memory T cell inflation: understanding cause and effect. Trends Immunol. 33, 84-90 (2012).

17. Holtappels, R., Pahl-Seibert, M. F., Thomas, D. \& Reddehase, M. J. Enrichment of immediate-early 1 (m123/pp89) peptide-specific CD8 T cells in a pulmonary CD62L(lo) memory-effector cell pool during latent murine cytomegalovirus infection of the lungs. J. Virol. 74, 11495-11503 (2000).

18. Torti, N., Walton, S. M., Brocker, T., Rulicke, T. \& Oxenius, A. Non-hematopoietic cells in lymph nodes drive memory CD8 T cell inflation during murine cytomegalovirus infection. PLoS Pathog. 7, e1002313 (2011).

19. Dekhtiarenko, I. et al. Peptide processing is critical for T-cell memory inflation and may be optimized to improve immune protection by CMV-based vaccine vectors. PLoS Pathog. 12, e1006072 (2016)

20. Hutchinson, $S$. et al. A dominant role for the immunoproteasome in CD8+ T cell responses to murine cytomegalovirus. PLOS ONE 6, e14646 (2011).

21. Hansen, S. G. et al. Profound early control of highly pathogenic SIV by an effector memory T-cell vaccine. Nature 473, 523-527 (2011).

22. Hansen, S. G. et al. Effector memory T cell responses are associated with protection of rhesus monkeys from mucosal simian immunodeficiency virus challenge. Nat. Med. 15, 293-299 (2009).

23. Klyushnenkova, E. N. et al. A cytomegalovirus-based vaccine expressing a single tumor-specific CD8 + T-cell epitope delays tumor growth in a murine model of prostate cancer. J. Immunother. 35, 390-399 (2012).
24. Qiu, Z. et al. Cytomegalovirus-based vaccine expressing a modified tumor antigen induces potent tumor-specific CD8(+) T-cell response and protects mice from melanoma. Cancer Immunol. Res. 3, 536-546 (2015).

25. Tsuda, Y. et al. A replicating cytomegalovirus-based vaccine encoding a single Ebola virus nucleoprotein CTL epitope confers protection against Ebola virus. PLoS Negl. Trop. Dis. 5, e1275 (2011).

26. Tsuda, Y. et al. A cytomegalovirus-based vaccine provides long-lasting protection against lethal Ebola virus challenge after a single dose. Vaccine 33, 2261-2266 (2015).

27. Welten, S. P. M., Baumann, N. S. \& Oxenius, A. Fuel and brake of memory T cell inflation. Med. Microbiol. Immunol. 208, 329-338 (2019).

28. Ynga-Durand, M. A., Dekhtiarenko, I. \& Cicin-Sain, L. Vaccine vectors harnessing the power of Cytomegaloviruses. Vaccines (Basel) 7, 152 (2019) https://doi.org/ $10.3390 /$ vaccines7040152.

29. Beyranvand Nejad, E. et al. Demarcated thresholds of tumor-specific CD8 T cells elicited by MCMV-based vaccine vectors provide robust correlates of protection. J. Immunother. Cancer 7, 25 (2019).

30. Smith, C. J., Turula, H. \& Snyder, C. M. Systemic hematogenous maintenance of memory inflation by MCMV infection. PLoS Pathog. 10, e1004233 (2014).

31. Takamura, S. et al. Specific niches for lung-resident memory CD8+ T cells at the site of tissue regeneration enable CD69-independent maintenance. J. Exp. Med. 213, 3057-3073 (2016).

32. Baumann, N. S. et al. Tissue maintenance of CMV-specific inflationary memory T cells by IL-15. PLoS Pathog. 14, e1006993 (2018).

33. Morabito, K. M. et al. Memory inflation drives tissue-resident memory CD8(+) T cell maintenance in the lung after intranasal vaccination with murine Cytomegalovirus. Front. Immunol. 9, 1861 (2018).

34. Morabito, K. M. et al. Intranasal administration of RSV antigen-expressing MCMV elicits robust tissue-resident effector and effector memory CD8+ T cells in the lung. Mucosal Immunol. 10, 545-554 (2017).

35. Zheng, X. et al. Mucosal CD8 $+\mathrm{T}$ cell responses induced by an MCMV based vaccine vector confer protection against influenza challenge. PLoS Pathog. 15 e1008036 (2019).

36. Pizzolla, A. et al. Resident memory CD8+ T cells in the upper respiratory tract prevent pulmonary influenza virus infection. Sci. Immunol. 2, eaam6970 (2017) https://doi.org/10.1126/sciimmunol.aam6970.

37. Slutter, B. et al. Dynamics of influenza-induced lung-resident memory $T$ cells underlie waning heterosubtypic immunity. Sci. Immunol. 2, eaag2031 (2017) https://doi.org/10.1126/sciimmunol.aag2031.

38. Goulding, J. et al. CD8 T cells use IFN-gamma to protect against the lethal effects of a respiratory poxvirus infection. J. Immunol. 192, 5415-5425 (2014).

39. Nie, S., Cornberg, M. \& Selin, L. K. Resistance to vaccinia virus is less dependent on TNF under conditions of heterologous immunity. J. Immunol. 183, 6554-6560 (2009).

40. Beura, L. K. et al. Intravital mucosal imaging of $\mathrm{CD} 8(+)$ resident memory $\mathrm{T}$ cells shows tissue-autonomous recall responses that amplify secondary memory. Nat. Immunol. 19, 173-182 (2018)

41. Park, S. L. et al. Local proliferation maintains a stable pool of tissue-resident memory T cells after antiviral recall responses. Nat. Immunol. 19, 183-191 (2018).

42. Moran, A. E. et al. T cell receptor signal strength in Treg and iNKT cell development demonstrated by a novel fluorescent reporter mouse. J. Exp. Med. 208, 1279-1289 (2011).

43. Osborn, J. F. et al. Enzymatic synthesis of core 2 O-glycans governs the tissuetrafficking potential of memory CD8(+) T cells. Sci. Immunol. 2, eaan6049 (2017) https://doi.org/10.1126/sciimmunol.aan6049.

44. Thomsen, A. R., Nansen, A., Madsen, A. N., Bartholdy, C. \& Christensen, J. P. Regulation of $\mathrm{T}$ cell migration during viral infection: role of adhesion molecules and chemokines. Immunol. Lett. 85, 119-127 (2003).

45. Wu, $\mathrm{T}$. et al. Lung-resident memory CD8 T cells (TRM) are indispensable for optimal cross-protection against pulmonary virus infection. J. Leukoc. Biol. 95, 215-224 (2014)

46. Redeker, A., Welten, S. P. \& Arens, R. Viral inoculum dose impacts memory T-cell inflation. Eur. J. Immunol. 44, 1046-1057 (2014).

47. Snyder, C. M. et al. Memory inflation during chronic viral infection is maintained by continuous production of short-lived, functional T cells. Immunity 29, 650-659 (2008).

48. Gordon, C. L. et al. Induction and maintenance of CX3CR1-intermediate peripheral memory CD8(+) T cells by persistent viruses and vaccines. Cell Rep. 23, 768-782 (2018)

49. Collins, P. L. \& Graham, B. S. Viral and host factors in human respiratory syncytial virus pathogenesis. J. Virol. 82, 2040-2055 (2008).

50. Quantius, J. et al. Influenza virus infects epithelial stem/progenitor cells of the distal lung: impact on Fgfr2b-driven epithelial repair. PLoS Pathog. 12, e1005544 (2016).

51. Lambert Emo, K. et al. Live imaging of influenza infection of the trachea reveals dynamic regulation of CD8+ T cell motility by antigen. PLoS Pathog. 12, e1005881 (2016) 
52. Salek-Ardakani, S. et al. The TNFR family members OX40 and CD27 link viral virulence to protective T cell vaccines in mice. J. Clin. Investig. 121, 296-307 (2011).

53. Hoekstra, M. E. et al. Long-distance modulation of bystander tumor cells by CD8(+) T cell-secreted IFNgamma. Nat. Cancer 1, 291-301 (2020).

54. Schenkel, J. M., Fraser, K. A., Vezys, V. \& Masopust, D. Sensing and alarm function of resident memory CD8(+) T cells. Nat. Immunol. 14, 509-513 (2013).

55. Jeyanathan, $M$. et al. CXCR3 signaling is required for restricted homing of parenteral tuberculosis vaccine-induced $T$ cells to both the lung parenchyma and airway. J. Immunol. 199, 2555-2569 (2017).

56. Slutter, B., Pewe, L. L., Kaech, S. M. \& Harty, J. T. Lung airway-surveilling CXCR3(hi) memory $\mathrm{CD} 8(+) \mathrm{T}$ cells are critical for protection against influenza $\mathrm{A}$ virus. Immunity 39, 939-948 (2013).

57. Abboud, G. et al. Tissue-specific programming of memory CD8 T cell subsets impacts protection against lethal respiratory virus infection. J. Exp. Med. 213, 2897-2911 (2016)

58. Gilchuk, P. et al. A distinct lung-interstitium-resident memory CD8(+) T cell subset confers enhanced protection to lower respiratory tract infection. Cell Rep. 16, 1800-1809 (2016).

59. Sakai, S. et al. Cutting edge: control of Mycobacterium tuberculosis infection by a subset of lung parenchyma-homing CD4 T cells. J. Immunol. 192, 2965-2969 (2014).

60. Groom, J. R. et al. CXCR3 chemokine receptor-ligand interactions in the lymph node optimize CD4+ T helper 1 cell differentiation. Immunity 37, 1091-1103 (2012).

61. Jenkins, M. R., Webby, R., Doherty, P. C. \& Turner, S. J. Addition of a prominent epitope affects influenza A virus-specific CD8+ T cell immunodominance hierarchies when antigen is limiting. J. Immunol. 177, 2917-2925 (2006).

62. Dekhtiarenko, I., Jarvis, M. A., Ruzsics, Z. \& Cicin-Sain, L. The context of gene expression defines the immunodominance hierarchy of cytomegalovirus antigens. J. Immunol. 190, 3399-3409 (2013).
63. Welten, S. P. et al. The viral context instructs the redundancy of costimulatory pathways in driving CD8(+) T cell expansion. Elife 4, e07486 (2015) https://doi. org/10.7554/eLife.07486.

64. Zurbach, K. A., Moghbeli, T. \& Snyder, C. M. Resolving the titer of murine cytomegalovirus by plaque assay using the M2-10B4 cell line and a low viscosity overlay. Virol. J. 11, 71 (2014).

65. Hickman, H. D. et al. Direct priming of antiviral CD8+ T cells in the peripheral interfollicular region of lymph nodes. Nat. Immunol. 9, 155-165 (2008).

66. Mercer, J. \& Helenius, A. Vaccinia virus uses macropinocytosis and apoptotic mimicry to enter host cells. Science 320, 531-535 (2008).

67. Beerli, C. et al. Vaccinia virus hijacks EGFR signalling to enhance virus spread through rapid and directed infected cell motility. Nat. Microbiol. 4, 216-225 (2019).

68. Altman, J. D. et al. Phenotypic analysis of antigen-specific T lymphocytes. Science 274, 94-96 (1996).

(i) Open Access This article is licensed under a Creative Commons Attribution 4.0 International License, which permits use, sharing, adaptation, distribution and reproduction in any medium or format, as long as you give appropriate credit to the original author(s) and the source, provide a link to the Creative Commons license, and indicate if changes were made. The images or other third party material in this article are included in the article's Creative Commons license, unless indicated otherwise in a credit line to the material. If material is not included in the article's Creative Commons license and your intended use is not permitted by statutory regulation or exceeds the permitted use, you will need to obtain permission directly from the copyright holder. To view a copy of this license, visit http://creativecommons. org/licenses/by/4.0/.

(c) The Author(s) 2021 